Interfaces and Free Boundaries 14 (2012), 431-454

DOI $10.4171 / \mathrm{IFB} / 287$

\title{
A nested variational time discretization for parametric Willmore flow
}

\author{
NADINE BALZANI \\ Institute for Numerical Simulation, Universität Bonn, Endenicher Allee 60, 53115 Bonn, Germany \\ E-mail:nadine.balzani@googlemail.com \\ MARTIN RUMPF \\ Institute for Numerical Simulation, Universität Bonn, Endenicher Allee 60, 53115 Bonn, Germany \\ E-mail:martin.rumpf@ins.uni-bonn.de
}

[Received 22 December 2010 and in revised form 3 July 2012]

\begin{abstract}
A novel variational time discretization of isotropic and anisotropic Willmore flow combined with a spatial parametric finite element discretization is applied to the evolution of polygonal curves and triangulated surfaces. In the underlying natural approach for the discretization of gradient flows a nested optimization problem has to be solved at each time step. Thereby, an outer variational problem reflects the time discretization of the actual Willmore flow and involves an approximate $L^{2}$-distance between two consecutive time steps and a fully implicit approximation of the Willmore energy. The mean curvature needed to evaluate the integrant of the latter energy is replaced by the time discrete, approximate speed from an inner, fully implicit variational scheme for mean curvature motion. To solve the resulting PDE constrained optimization problem at every time step duality techniques from PDE optimization are applied. Computational results underline the robustness of the new scheme, in particular with respect to large time steps, and show applications to surface restoration and blending.
\end{abstract}

2010 Mathematics Subject Classification: Primary 65M60; Secondary 53C44.

Keywords: Willmore flow, geometric evolution problem, finite element discretization, curvature flow

\section{Introduction}

This paper presents a new variational scheme for the time and space discretization of parametric isotropic and anisotropic Willmore flow.

Willmore flow is defined as the geometric gradient flow of the Willmore energy with respect to the $L^{2}$-metric, where the Willmore energy for a $d$-dimensional surface $m$ embedded in $\mathbb{R}^{m}$ with $m \geqslant d+1$ is defined as

$$
w[x]:=\frac{1}{2} \int_{m[x]} \mathbf{h}^{2} \mathrm{~d} a,
$$

with $x$ denoting a parametrization of $m=m[x]$, $\mathbf{h}$ the mean curvature, and the $L^{2}$-metric

$$
g_{x}\left(v_{1}, v_{2}\right)=\int_{m[x]} v_{1} v_{2} \mathrm{~d} a
$$

is defined on variations $x+v_{i} n$ of the surface $m$ in the direction of the surface normal $n$. Here, the mean curvature $\mathbf{h}$ itself represents the $L^{2}$-gradient of the area functional $a[x]=\int_{m} \mathrm{~d} a$ and equals 
the sum of the principal curvatures. In the hyper-surface case $(m=d+1)$ Willmore flow leads to the fourth order parabolic evolution problem

$$
\partial_{t} x=\Delta m \mathbf{h} n+\mathbf{h}\left(|S|_{2}^{2}-\frac{1}{2} \mathbf{h}^{2}\right) n
$$

which defines, for a given initial surface $m_{0}$, a family of surfaces $m(t)$ for $t \geqslant 0$ with $m(0)=m_{0}$ $[31,49,51]$. Here, $\Delta m$ is the Laplace Beltrami operator on a surface $m, S$ denotes the shape operator or Weingarten map on $m$ encoding the variation of the normal as an endomorphism on the tangent space, and $|\cdot|_{2}$ the Frobenius norm on the space of endomorphisms on the tangent bundle $5 \mathrm{~m}$. Applications of a minimization of Willmore energy and of Willmore flow evolution as the corresponding gradient flow include the modeling of edge sets in imaging [10, 36, 38, 53], surface modeling [5, 6, 50,52] and extending the Willmore energy to the Helfrich energy in the mathematical treatment of biological membranes [20, 29, 48]. The analytic treatment of the Willmore flow was investigated by Polden [43, 44]. Sharp estimates on long time existence and regularity were obtained by Kuwert and Schätzle [31, 32]. Willmore flow of curves has been studied by Dziuk, Kuwert, and Schätzle in [26]. Recently, Rivière [46] extended results of Kuwert and Schätzle [33] from co-dimension 3 to arbitrary co-dimensions. He proved a weak compactness result for Willmore surfaces with energy less than $16 \pi$ and a strong compactness of Willmore tori below the energy level $16 \pi$. He also provided a new formulation for the weak Euler-Lagrange equation of the Willmore functional for immersed surfaces in $\mathbb{R}^{m}$. The numerical results of Mayer and Simonett [35] suggest that the above estimate is optimal in the sense that the flow develops a singularity if the initial surface has energy greater than $16 \pi$.

In Finsler geometry the focus is on anisotropic area measurement encoded in the functional $a_{\gamma}[x]=\int_{m} \gamma(n) \mathrm{d} a$ where the local area weight $\gamma(n)$ depends on the surface orientation via a positive 1-homogeneous anisotropy function $\gamma$. Now, in analogy to the isotropic case the anisotropic mean curvature $\mathbf{h}_{\gamma}$ is defined as the $L^{2}$-gradient of the anisotropic area $a_{\gamma}[\cdot]$ and can be evaluated as $\mathbf{h}_{\gamma}=\operatorname{div} m\left(\gamma_{z}(n)\right)$. Hence, the anisotropic Willmore functional is given by $w_{\gamma}[x]=\frac{1}{2} \int_{m} \mathbf{h}_{\gamma}^{2} \mathrm{~d} a$. Clarenz [12] has shown that Wulff shapes are the only minimizers in the class of immersions of twodimensional surface $m$ into $\mathbb{R}^{3}$. Recently, Bellettini \& Mugnai [3] investigated the first variation of this functional in the smooth case. Clarenz [11] and Palmer [41, 42] studied variational problems involving anisotropic bending energies for surfaces with and without boundaries. Anisotropic Willmore flow of hyper-surfaces can be rephrased as the following parabolic fourth order PDE

$$
\partial_{t} x=\Delta_{\gamma} \mathbf{h}_{\gamma} n+\mathbf{h}_{\gamma}\left(|S|_{\gamma}^{2}-\frac{1}{2} \mathbf{h} \mathbf{h}_{\gamma}\right) n
$$

where $\Delta_{\gamma}:=\operatorname{div} m\left(\gamma_{z z}(n) \nabla_{m}\right)$ is the generalized Laplace-Beltrami operator with divm and $\nabla_{m}$ being the tangential divergence and gradient, respectively. Furthermore, $|S|_{\gamma}^{2}=\operatorname{tr}\left(\gamma_{z z}(n) S^{2}\right)$ is the weighted, squared Frobenius norm of the shape operator, and $\gamma_{z z}=D^{2} \gamma$ the second derivatives of the anisotropy function $\gamma$.

Concerning the numerical approximation of Willmore flow in the isotropic case, Rusu [47] proposed a mixed method for the surface parametrization $x$ and the mean curvature vector $\mathbf{h} n$ as independent variables, see also [13] for an application to surface restoration. A level set formulation in the hyper-surface case was presented in [19]. Deckelnick and Dziuk [14] investigated the convergence of a space discrete related scheme in the case of graph surfaces and Deckelnick and Schieweck demonstrated convergence of a conforming finite element approximation for axial 
symmetric surfaces [16]. An error analysis in the case of the elastic flow of curves was recently presented by Dziuk and Deckelnick in [15]. Furthermore, we refer to Barrett, Garcke and Nürnberg [1], Bobenko and Schröder [7] and Dziuk [25] for alternative numerical methods for Willmore flow on triangulation surfaces. Diewald [17] has extended the approach of Rusu [47] to the parametric anisotropic Willmore flow for closed surfaces and Droske [18] and Nemitz [37] investigated a level set discretization. In [45] Pozzi discussed the Willmore flow of curves in higher co-dimension. The time discretization of the anisotropic mean curvature flow has been considered by Dziuk already in $[23,24]$ and he gave convergence results for curves.

Contrasting with fully explicit time discretizations - not mentioned here - the above numerical approaches are all characterized by some type of semi-implicit time discretization and require the solution of linear systems of equations at each time step. Roughly speaking geometric differential operators are assembled at the previous time step. Even though discrete energy estimates exist in many cases one observes practical restrictions on the time step size. This shortcoming motivated the development of a new concept for the time discretization of Willmore flow leveraging the variational time discretization of general gradient flows (first preliminary results on this approach have been presented in the proceedings article [40]). Given an energy $e[\cdot]$ on a (in general infinite dimensional) manifold with metric $g$ and the gradient flow $\dot{x}=-\operatorname{grad}_{g} e[x]$ with initial data $x^{0}$ one defines a sequence of time discrete solutions $\left(x^{k}\right)_{k=0, \cdots}$ with $x_{k} \approx x(k \tau)$ for the time step size $\tau$ via a variational problem, to be solved in each time step, i.e.,

$$
x^{k+1}=\arg \min _{x} \operatorname{dist}\left(x, x^{k}\right)^{2}+2 \tau e[x],
$$

where $\operatorname{dist}\left(x, x^{k}\right)=\inf _{\gamma \in \Gamma\left[x^{k}, x\right]} \int_{0}^{1} \sqrt{g_{\gamma(s)}(\dot{\gamma}(s), \dot{\gamma}(s))} \mathrm{d} s$ is the Riemannian distance on the manifold from $x$ to $x_{k}$ defined as the length of the shortest path, $\Gamma\left[x^{k}, x\right]$ denotes the set of smooth curves $\gamma$ with $\gamma(0)=x^{k}$ and $\gamma(1)=x$. As an immediate consequence, one obtains the energy estimate $e\left[x^{k+1}\right]+\frac{1}{2 \tau} \operatorname{dist}\left(x^{k+1}, x^{k}\right)^{2} \leqslant e\left[x^{k}\right]$. For geometric problems, this approach has already been considered by Luckhaus and Sturzenhecker [34] in the case of mean curvature motion via a fully implicit variational time discretization in $B V$. Chambolle [8] investigated a reformulation of this approach in terms of a level set method. A related method for anisotropic mean curvature motion is discussed in $[2,9]$.

Here, we build upon this concept and proceed as follows in the case of Willmore flow. We aim at balancing the squared distance of the unknown surface at time $t_{k+1}=t_{k}+\tau$ from the current surface at time $t_{k}$ and a suitable approximation of the Willmore energy at time $t_{k+1}$ scaled by twice the time step size. Solving a fully implicit time discrete problem for mean curvature motion for the unknown surface at time $t_{k+1}$, we can regard the resulting discrete speed of evolution as a time discrete, fully implicit approximation of the mean curvature vector. This approximate mean curvature vector is then used to approximate the Willmore functional. Thus, we resort a nested minimization problem within each time step, where an inner problem solves for an implicit mean curvature vector, while an outer problem reflects an actual implicit, variational formulation for a time step of Willmore flow. As it will be discussed in detail the resulting nested time discretization experimentally turns out to be unconditionally stable and effectively allows for time steps of the order of the spatial grid size. Furthermore, the approach can be generalized to anisotropic Willmore flow.

The paper is organized as follows. In Section 2 we derive time discretizations for isotropic Willmore flow of compact surfaces and for surfaces with boundaries on which $C^{1}$ boundary conditions are prescribed. Furthermore, we generalize the approach to anisotropic Willmore flow. 
Then in Section 3 we discuss a fully discrete numerical scheme based on piecewise affine finite elements on simplicial surface meshes. In Section 4 the duality technique from PDE constraint optimization is revisited to develop suitable minimization algorithms for the optimization problems to be solved in each time step. Finally, in Section 5 computational results are presented. An appendix collects essential ingredients of the corresponding algorithms to facilitate the implementation.

\section{Nested time discretization of Willmore flow}

In this section we will apply the concept of natural time discretization to Willmore flow and derive a nested (but still spatially continuous) variational problem for each time step. To begin with, we recall the corresponding time discretization of mean curvature motion. Let us consider a surface $m=$ $m[x]$, where $x$ indicates a parametrization of $m$ and can also be considered as the identity map on $m$ parametrizing $m$ over itself. Following the above abstract approach, we ask for the next time step $m[y]$ with a corresponding parametrization $y=y[x]$ given as the minimizer of the functional $\operatorname{dist}(m[y], m[x])^{2}+2 \tilde{\tau} \int_{m[y]} \mathrm{d} a$, where $\tilde{\tau}$ denotes the time step size. Here, we restrict ourselves to surfaces pairs $m[y], m[x]$ which allow a unique graph representation $x \mapsto x+f(x) n(x)$, $y \mapsto y+\tilde{f}(y) n(y)$ of $m[y]$ over $m[x]$ and of $m[x]$ over $m[y]$, respectively. Furthermore, we define $\operatorname{dist}(m[y], m[x])=\left(\int_{\mathfrak{m}[x]} f(x)^{2} \mathrm{~d} a+\int_{m[y]} \tilde{f}(y)^{2} \mathrm{~d} a\right)^{\frac{1}{2}}$ as the $L^{2}$-distance between surfaces, and $\int_{m[y]} \mathrm{d} a$ is the surface area of $m[y]$. To define the $L^{2}$ distance between such surfaces, we take into account the straightforward linearization argument

$$
2 \int_{\mathfrak{m}[y]} \mathrm{d} a=(2-d) \int_{\mathfrak{m}[x]} \mathrm{d} a+\int_{\mathfrak{m}[x]}\left|\nabla_{m[x]} y\right|^{2} \mathrm{~d} a+O\left(\|y-x\|_{C^{1}(m[x])}^{2}\right)
$$

for a parametrization $y$ which are $C^{1}$ close to $x$ (here $\nabla_{\mathfrak{m}[x]} y$ is defined as the tangential gradient of $y$ with respect to the surface $m[x]$, and $\|f\|_{C^{1}(m[x])}$ denotes the $C^{1}$ norm of $f$ on $m[x]$. For the proof of (2.1) we refer to Appendix A. Furthermore, observing that the minimization can be restricted to surface parametrizations $y$ for which $y-x \perp m[x]$ we obtain the following approximate variational problem for a single time step of mean curvature motion:

Given a surface $m[x]$ parameterized by a mapping $x$ we ask for a mapping $y=y[x]$ which minimizes the functional

$$
e[x, y]:=\int_{m[x]}(y-x)^{2}+\tilde{\tau}\left|\nabla_{m[x]} y\right|^{2} \mathrm{~d} a .
$$

The resulting weak form of the corresponding Euler-Lagrange equation is

$$
0=\int_{m[x]}(y-x) \cdot \theta+\tilde{\tau} \nabla_{\mathfrak{m}[x]} y: \nabla_{m[x]} \theta \mathrm{d} a
$$

for smooth test functions $\theta$ defined on $m[x]$, where $A: B=\operatorname{tr}\left(A^{T} B\right)$. Obviously, this equation coincides with the classical scheme for a single semi-implicit time step of mean curvature motion proposed by Dziuk in [22]. 


\subsection{Willmore flow on closed surfaces}

The abstract variational time discretization of isotropic Willmore flow reads as follows

$$
\operatorname{dist}\left(m[x], m\left[x^{k}\right]\right)^{2}+\tau \int_{m[x]} \mathbf{h}^{2} \mathrm{~d} a \rightarrow \min .
$$

For the first term we consider the same approximation as above for mean curvature motion. To verify that this natural discretization is consistent for smooth surfaces with the well-known fourth order parabolic equation (1.1) we combine the above insight for mean curvature motion on the derivation of the parabolic term with the derivation of the PDE from the variational principle in [19]. For the Willmore energy, we now make use of the following observation. By definition the mean curvature $\mathbf{h}=\mathbf{h}[x]$ is the $L^{2}$-gradient of the area functional on a surface $m[x]$ and mean curvature motion is the corresponding gradient flow. Hence, the mean curvature vector $\mathbf{h}[x] n[x]$ with $n=n[x]$ denoting the normal on $m[x]$ can be approximated by the difference quotient in time $\frac{y[x]-x}{\tilde{\tau}}$, where $y[x]$ is the minimizer of $e[x, \cdot]$ in (2.2) for time step size $\tilde{\tau}$. Thus, for small $\tilde{\tau}$ the functional $\frac{1}{2} \int_{m[x]} \frac{(y[x]-x)^{2}}{\tilde{\tau}^{2}} \mathrm{~d} a$ is an approximation the Willmore functional on $m[x]$.

This enables us to derive an approximate variational time discretization of Willmore flow, which does not require the explicit evaluation of the mean curvature on the unknown surface $m[y]$ at the next time step. In fact, for a given surface parametrization $x^{k}$ of the surface $m\left[x^{k}\right]$ at a time step $k$ we define the functional

$$
w\left[x^{k}, x, y\right]:=\int_{m\left[x^{k}\right]}\left(x-x^{k}\right)^{2} \mathrm{~d} a+\frac{\tau}{\tilde{\tau}^{2}} \int_{m[x]}(y-x)^{2} \mathrm{~d} a,
$$

where we select $y=y[x]$ as the minimizer of (2.2) with time step size $\tilde{\tau}$. Finally, we obtain the following time discretization of Willmore flow:

Given an initial surface $m\left[x^{0}\right]$ with parametrization $x^{0}$ we define a sequence of surfaces m $\left[x^{k}\right]$ with parametrizations $x^{k}$ for $k=1, \ldots$ via the solution of the following sequence of nested variational problem

$$
\begin{aligned}
x^{k+1} & =\arg \min _{x} w\left[x^{k}, x, y[x]\right], \text { where } \\
y[x] & =\arg \min _{y} e[x, y] .
\end{aligned}
$$

Thereby, the inner variational problem (2.5) is quadratic. Thus, the resulting Euler-Lagrange equation (2.3) is a linear elliptic PDE and we end up with a PDE constrained optimization problem for each time step.

To be more explicit, let us examine circles in the plane. Under Willmore flow circles expand according to the ODE $\dot{R}(t)=\frac{1}{2} R(t)^{-3}$ for the radius. In comparison to this we obtain for the nested variational time discretization with a slight misuse of notation

$$
w\left[R_{k}, R, \tilde{R}\right]=2 \pi R_{k}(R-\tilde{R})^{2}+2 \pi R \tau \frac{(R-\tilde{R})^{2}}{\tilde{\tau}^{2}}, \quad e[R, \tilde{R}]=2 \pi R\left((R-\tilde{R})^{2}+\tilde{\tau} \frac{\tilde{R}^{2}}{R^{2}}\right),
$$


for a time discrete radii $R_{k}, R$, and $\tilde{R}$. Thus, by a straightforward computation we deduce the nonlinear equation $\frac{R-R_{k}}{\tau}=\frac{1}{2} \frac{R^{4}-3 R^{2} \tilde{\tau}}{\left(R^{2}+\tilde{\tau}\right)^{3} R_{k}}$ to be solved for $R$ as the radius at the next time step. This is indeed an implicit first order scheme for the above ODE. For a discussion of the consistency of this variational approach in the general case we also refer to [27], where the scheme is applied in the context of phase fields.

\subsection{Willmore flow with boundary conditions}

Next, with the application to surface restoration in mind let us consider the case of surfaces $m[x]$ with boundary $\Gamma[x]=\partial m[x]$ and aim at prescribing boundary conditions both for $x$ and for the normal $n[x]$ - at least in an approximate sense - on $\Gamma[x]$. Therefore, we modify the inner minimization problem and consider an energy

$$
e^{\partial}[x, y]=\int_{m[x]}(y-x)^{2}+\tilde{\tau}\left|\nabla_{m[x]} y\right|^{2} \mathrm{~d} a-2 \tilde{\tau} \int_{\Gamma[x]} n^{c o} \cdot y \mathrm{~d} s
$$

for given $x$ and $n^{c o}$, where $n^{c o}$ is the co-normal perpendicular to $n$ and to the boundary $\partial m$. Indeed, for smooth surface $m$ and smooth boundary $\Gamma[x]$ prescribing the co-normal is up to the direction equivalent to prescribing the surface normal. The resulting weak form of the Euler Lagrange equation for fixed $x$ and a minimizer $y[x]$ of $e^{\partial}[x, \cdot]$ in (2.6) is

$$
0=\int_{m[x]} \frac{y-x}{\tilde{\tau}} \cdot \psi+\nabla_{m[x]} y: \nabla_{m[x]} \psi \mathrm{d} a-\int_{\partial m[x]} n^{c o} \cdot \psi \mathrm{d} s
$$

for all test functions $\psi \in C^{\infty}(\bar{m}[x])$. Hence, we obtain the time discrete mean curvature motion equation $\frac{y-x}{\tilde{\tau}}=\Delta m[x] y$ on $m[x]$ and the boundary condition $\partial_{n^{c o}[x]} y=n^{c o}$ on $\Gamma[x]$, where $n^{c o}[x]$ denotes the actual co-normal of $m[x]$. If we assume that $y[x]-x$ converges to 0 in $C^{1}$ for $\tilde{\tau} \rightarrow 0$ where $x$ is the minimizer of the energy $w\left[x^{k}, x, y\right]$ and $y[x]$ minimizes $e^{\partial}[x, y]$, then we observe that

$$
\partial_{n^{c o}[x]} y=\nabla_{m[x]} y \cdot n^{c o}[x] \rightarrow \nabla_{m[x]} x \cdot n^{c o}[x]=n^{c o}[x]
$$

for $\tilde{\tau} \rightarrow 0$. Therefore, we obtain the approximate boundary condition $n^{c o}[x]=n^{c o}+o(1)$ on $\Gamma[x]$ with the usual Landau symbol $o(1) \rightarrow 0$ for $\tau \rightarrow 0$.

Alternatively, in particular in the context of surface restoration, we may assume that $m[x]$ is the varying part of a larger surface $\widetilde{m}[x]$ with an outer part $m^{\text {ext }}$ being fixed. Correspondingly, the parametrization $x$ is extended to some parametrization $x^{\text {ext }}$ outside of $m[x]$. As before $\Gamma[x]$ is supposed to denote the boundary of $m[x]$. Then, we again reformulate the inner minimization problem and take into account the energy

$$
\tilde{e}^{\partial}[x, y]=\int_{\tilde{m}[x]}(y-x)^{2}+\tilde{\tau}\left|\nabla_{m}[x] y\right|^{2} \mathrm{~d} a,
$$

which coincides with the original energy $e[x, y]$ except that it is now integrated over the whole surface $\widetilde{m}[x]$ and $y$ is allowed to vary on $\widetilde{m}[x]$. In this case we obtain as an Euler Lagrange 
condition $\partial_{n^{c o}[x]} y[x]-\partial_{n^{c o}\left[x^{\mathrm{ex} t}\right]} y[x]=0$ on $\Gamma[x]$. Here, $n^{c o}\left[x^{\mathrm{ext}}\right]$ is the outer co-normal of $m^{\mathrm{ext}}$ on $\Gamma[x]$. If, we assume as above that $y[x]-x \rightarrow 0$ for $\tilde{\tau} \rightarrow 0$, then

$$
\begin{aligned}
\partial_{n^{c o}[x]} y[x] \rightarrow \partial_{n^{c o}[x]} x & =n^{c o}[x], \quad \text { and } \\
\partial_{n^{c o}\left[x^{\mathrm{ext}}\right]} y[x] \rightarrow \partial_{n^{c o}[\mathrm{ext}]} x^{\mathrm{ext}} & =n^{c o}\left[x^{\mathrm{ext}}\right] .
\end{aligned}
$$

Hence, once again, $n^{c o}[x]=n^{c o}\left[x^{\mathrm{ext}}\right]+o(1)$ on $\Gamma[x]$ with $o(1) \rightarrow 0$ for $\tau \rightarrow 0$, where in this case the co-normal $n^{c o}\left[x^{\mathrm{ext}}\right]$ is induced by the prescribed outer surface $m\left[x^{\mathrm{ext}}\right]$.

\subsection{Anisotropic Willmore flow of closed surfaces}

Finally, let us investigate the time discretization of anisotropic Willmore flow in co-dimension 1 with $m=d+1$. The corresponding abstract variational time discretization reads as follows

$$
\operatorname{dist}\left(m[x], m\left[x^{k}\right]\right)^{2}+\tau \int_{m[x]} h_{\gamma}^{2} \mathrm{~d} a \rightarrow \min ,
$$

where $h_{\gamma}=\operatorname{div} m\left(n_{\gamma}\right)=\operatorname{div} m\left(\gamma_{z} \circ n\right)$ denotes the generalized mean curvature. This time we again replace the $L^{2}$-distance between the two manifolds $m[x]$ and $m\left[x^{k}\right]$ by $\int_{m[x]}|y-x|^{2} \mathrm{~d} a$ under the assumption of sufficient regularity of $x$ and $y$. Moreover, as in the isotropic case, we make use of the fact that the $L^{2}$-gradient of the anisotropic area functional is the generalized mean curvature and hence, the time discrete speed $\frac{y[x]-x}{\tilde{\tau}}$ extracted from a variational time discretization of anisotropic curvature motion approximates the generalized curvature vector $h_{\gamma}[x] n[x]$ and can be used to approximate the anisotropic Willmore energy. In the anisotropic case we do not consider a linearization of the variational approach for generalized curvature motion as in the isotropic approach, but we define $y[x]$ to be the minimizer of the nonlinear functional

$$
e_{\gamma}[x, y]=\int_{m[x]}(y-x)^{2} \mathrm{~d} a+2 \tilde{\tau} \int_{m[y]} \gamma(n[y]) \mathrm{d} a
$$

in $y$ for given parametrization $x$. Then, collecting the different building blocks we end up with the following fully nonlinear variational time discretization of anisotropic Willmore flow:

$$
\begin{aligned}
x^{k+1} & =\arg \min _{x} w\left[x^{k}, x, y[x]\right], \quad \text { where } \\
y[x] & =\arg \min _{y} e_{\gamma}[x, y] .
\end{aligned}
$$

Let us remark that this variational time discretization does not involve derivatives of the anisotropy. Nevertheless, as we will see below, differentiation is indispensable to set up a descent algorithm for this functional.

\section{Finite element discretization in space}

In this section we introduce a suitable space discretization based on piecewise linear finite elements. Here, we follow the guideline for finite elements on surfaces introduced by Dziuk [21]. Thus, we consider simplicial meshes $m[X]$ - polygonal curves for $d=1$ and triangulation surfaces for $d=2$ - as approximations of the $d$ dimensional surfaces $m[x]$. Thereby, $X$ is a parametrization of 
the simplicial mesh $m[X]$ which is, for a fixed mesh topology, uniquely described by a vector $\bar{X}$ of vertex positions of the mesh. To clarify the notation we will always denote discrete quantities with upper case letters to distinguish them from the corresponding continuous quantities in lower case letters. Furthermore, a bar on top of a discrete function indicates the corresponding nodal vector, i.e., $\bar{X}=\left(\bar{X}_{i}\right)_{i \in I}$, where $\bar{X}_{i}=\left(X_{i}^{1}, \cdots, X_{i}^{m}\right)$ is the coordinate vector of the $i$ th vertex of the mesh and $I$ denotes the index set of vertices. For $d=1$ each element $T$ of a polygonal curve is a line segment with nodes $X_{1}$ and $X_{2}$ (using local indices) and for $d=2$ elements $T$ of a triangulation are planar triangles with vertices $X_{0}, X_{1}$, and $X_{2}$ and edge vectors $F_{0}=X_{2}-X_{1}, F_{1}=X_{0}-X_{2}$, and $F_{2}=X_{1}-X_{0}$.

Given a simplicial surface $m[X]$ we denote by

$$
v(m[X]):=\left\{U \in C^{0}(m[X])|U|_{T} \in P_{1} \forall T \in m[X]\right\}
$$

the associated piecewise affine finite element space. With a slight misuse of notation the mapping $X$ itself is considered as an element in $V(\mathscr{m}[X])^{m}$. Let $\left\{\Phi_{i}\right\}_{i \in I}$ be the nodal basis of $\mathcal{V}(\mathfrak{m}[X])$. For $U \in V(m[X])$ we obtain $U=\sum_{i \in I} U\left(X_{i}\right) \Phi_{i}$ and $\bar{U}=\left(U\left(X_{i}\right)\right)_{i \in I}$ - in agreement with our previous definition as we recover $\bar{X}=\left(X_{i}\right)_{i \in I}$.

Next, let us introduce the mass matrix $M[X]$ and the stiffness matrix $L[X]$ of the discrete surface $m[X]$, whose entries are given by $M_{i j}[X]=\int_{m_{[X]}} \Phi_{i} \Phi_{j} \mathrm{~d} a$ and $L_{i j}[X]=\int_{m_{[X]}} \nabla_{m_{[X]}} \Phi_{i}$. $\nabla_{m_{[X]}} \Phi_{j} \mathrm{~d} a$. The corresponding block structured matrices, which act in an identical way simultaneously on the $m$ coordinates of a vector with $m$ components for each node, are denoted by $\mathbf{M}[X]$ and $\mathbf{L}[X]$, respectively. As usual all matrices are assembled from corresponding local matrices computed on the simplices $T$ of $m[X]$.

\subsection{Willmore flow of closed surfaces}

Now, we have all the ingredients at hand to derive the fully discrete nested time discretization of Willmore flow, as the spatially discrete counterpart of (2.4) and (2.5):

Given an discrete initial surface $m\left[X^{0}\right]$ with discrete parametrization $X^{0}$ we compute a sequence of surfaces $m\left[X^{k}\right]$ with parametrizations $X^{k}$ by solving the following nested finite dimensional variational problem:

$$
\begin{aligned}
X^{k+1} & =\arg \min _{X \in \mathcal{V}\left(m\left[X^{k}\right]\right)^{m}} W\left[X^{k}, X, Y[X]\right], \text { where } \\
Y[X] & =\arg \min _{Y \in \mathcal{V}(m[X])^{m}} E[X, Y] .
\end{aligned}
$$

Here, the discrete functionals are given by

$$
\begin{aligned}
E[X, Y] & :=\int_{m[X]}(Y-X)^{2}+\tilde{\tau}\left|\nabla_{m[X]} Y\right|^{2} \mathrm{~d} a \\
& =\mathbf{M}[X](\bar{Y}-\bar{X}) \cdot(\bar{Y}-\bar{X})+\tilde{\tau} \mathbf{L}[X] \bar{Y} \cdot \bar{Y} \\
W\left[X^{k}, X, Y\right]: & \int_{m\left[X^{k}\right]}\left(X-X^{k}\right)^{2} \mathrm{~d} a+\frac{\tau}{\tilde{\tau}^{2}} \int_{m[X]}(Y-X)^{2} \mathrm{~d} a \\
& =\mathbf{M}\left[X^{k}\right]\left(\bar{X}-\bar{X}^{k}\right) \cdot\left(\bar{X}-\bar{X}^{k}\right)+\frac{\tau}{\tilde{\tau}^{2}} \mathbf{M}[X](\bar{Y}-\bar{X}) \cdot(\bar{Y}-\bar{X})
\end{aligned}
$$



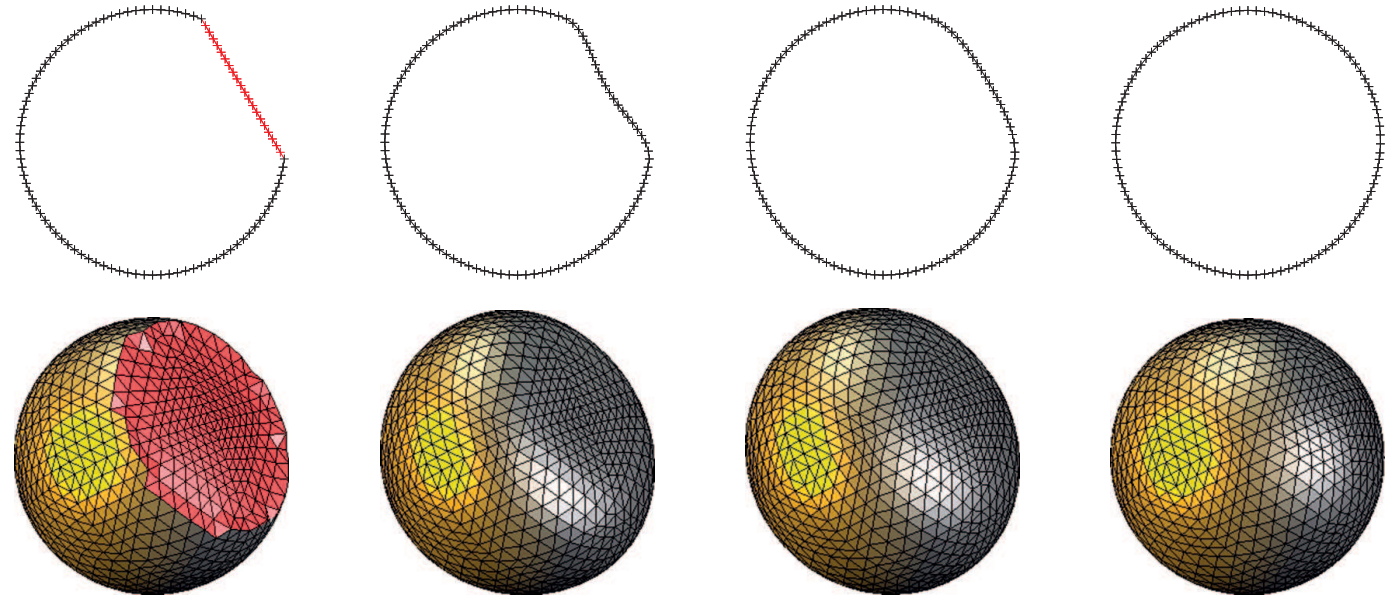

FIG. 1. Discrete isotropic Willmore flow is applied to a curve segment and a surface patch (plotted in red on the left at the initial time) with boundary conditions for the position and the co-normal. From left to right the solution together with the underlying triangulation is shown at time $0.0,0.02,0.45$, and 1.12 , where the underlying sphere radius is 1 and we have applied the time step size $\tau=h=0.02$.

as straightforward spatially discrete counterpart of the functionals $e[x, y]$ and $w\left[x^{k}, x, y\right]$, respectively. Analogously to the spatially continuous case in (2.3), for a given $X$, the nodal vector $\bar{Y}[X]$ solves the linear system of equation

$$
(\mathbf{M}[X]+\tilde{\tau} \mathbf{L}[X]) \bar{Y}[X]=\mathbf{M}[X] \bar{X} .
$$

Appendix B provides explicit formulas for the entries of the mass and stiffness matrices.

\subsection{Willmore flow with boundary conditions}

In the case of a discrete surface restoration application we consider a discrete simplicial surface $\widetilde{m}[X]$ consisting of the actual surface patch $m[X]-$ to be modified for instance in a surface restoration application - and a polygonal ring $m^{\text {ext }}[X]$ consisting of one layer of simplices around the discrete boundary $\Gamma[X]=\partial m[x]$ on which $X$ has a given fixed parametrization $X^{\text {ext }}$. We split the set of nodes $\eta$ of $m[X]$ into the set of interior nodes $\eta^{i n t}$ of $m[X]$, and boundary nodes $\eta^{\partial}$, and denote the corresponding index sets by $I, I^{i n t}$, and $I^{\partial}$, respectively. Let us emphasize that the nodal vectors for functions in $v_{0}(\widetilde{m})$ and in $V(m)$ can be identified because of the single layer assumption on $m^{\mathrm{ext}}[X]$. Furthermore, $v^{\text {int }}(m[X])$ denotes the subspace of $v(m[X])$ of functions vanishing on $\Gamma[X]$ and we introduce the obvious restriction operator $R: \mathbb{R}^{|I|} \rightarrow \mathbb{R}^{\left|I^{\text {int }}\right|}$ and a corresponding extension operators $E: \mathbb{R}^{\left|I^{i n t}\right|} \rightarrow \mathbb{R}^{|I|}$ with $(E \bar{U})_{i}=0$ for $i \in I^{\partial}$. The corresponding block operators acting on nodal vectors are denoted by $\mathbf{R}$ and $\mathbf{E}$, respectively. Hence, a valid nodal vector in $\mathbb{R}^{3|I|}$ can be written as $\mathbf{E} \bar{X}+\bar{X}^{\text {ext }}$, where $\bar{X}^{\text {ext }}$ is the vector with prescribed vertex positions at boundary nodes and zero entries for all interior nodes.

To prescribe a discrete co-normal we proceed as follows. On simplicial faces of $\Gamma[X]$ we compute $N^{c o}$ as the (piecewise constant) co-normal lying in the plane of the boundary elements 
of $m^{\text {ext }}[X]$ and define a corresponding (non unit length) nodal vector

$$
\bar{N}^{c o}[X]:=\left(\int_{\partial m[X]} N^{c o} \Phi_{i} \mathrm{~d} s\right)_{i \in I}
$$

in $\mathbb{R}^{d|I|}$. Then discrete counterpart $E^{\partial}[\cdot, \cdot]$ on $V^{i n t}\left(m\left[X^{k}\right]\right) \times V(m[X])$ of the functional $e^{\partial}[\cdot, \cdot]$ is given by

$$
\begin{aligned}
E^{\partial}[X, Y]:= & \int_{m[X]}(Y-X)^{2}+\tilde{\tau}\left|\nabla_{m[X]} Y\right|^{2} \mathrm{~d} a-2 \tilde{\tau} \int_{\partial m_{[X]}} N^{c o} \cdot Y \mathrm{~d} s \\
= & \mathbf{M}[X]\left(\bar{Y}-\mathbf{E} \bar{X}-\bar{X}^{\text {ext }}\right) \cdot\left(\bar{Y}-\mathbf{E} \bar{X}-\bar{X}^{\text {ext }}\right) \\
& +\tilde{\tau} \mathbf{L}[X] \bar{Y} \cdot \bar{Y}-2 \tilde{\tau} \bar{N}^{c o} \cdot \bar{Y}
\end{aligned}
$$

with $\bar{X} \in \mathbb{R}^{m\left|I^{i n t}\right|}$ and $\bar{X}^{\text {ext }}, \bar{Y} \in \mathbb{R}^{m|I|}$, whereas the corresponding functional $W\left[X^{k}, \cdot, \cdot\right]$ on the finite element space $v^{i n t}\left(\mathfrak{m}\left[X^{k}\right]\right) \times v(m[X])$ can be rephrased as

$$
\begin{aligned}
W^{\partial}\left[X^{k}, X, Y\right]:= & \int_{m\left[X^{k}\right]}\left(X-X^{k}\right)^{2} \mathrm{~d} a+\frac{\tau}{\tilde{\tau}^{2}} \int_{m[X]}(Y-X)^{2} \mathrm{~d} a \\
= & \mathbf{M}\left[X^{k}\right]\left(\mathbf{E} \bar{X}-\bar{X}^{k}\right) \cdot\left(\mathbf{E} \bar{X}-\bar{X}^{k}\right) \\
& +\frac{\tau}{\tilde{\tau}^{2}} \mathbf{M}[X]\left(\bar{Y}-\mathbf{E} \bar{X}-\bar{X}^{\text {ext }}\right) \cdot\left(\bar{Y}-\mathbf{E} \bar{X}-\bar{X}^{\text {ext }}\right) .
\end{aligned}
$$

Hence, to compute $\bar{Y}[X]$ we have to solve the linear system of equation

$$
(\mathbf{M}[X]+\tilde{\tau} \mathbf{L}[X]) \bar{Y}[X]=\mathbf{M}[X]\left(\mathbf{E} \bar{X}-\bar{X}^{e x t}\right)+\tilde{\tau} \bar{N}^{c o} .
$$

In the case of the alternative approach, one defines the discrete functional $\widetilde{E}^{\partial}[\cdot, \cdot]$ on $V^{i n t}\left(m\left[X^{k}\right]\right) \times$ $v_{0}(\widetilde{m}[X])$ by

$$
\begin{aligned}
\widetilde{E}^{\partial}[X, Y] & :=\int_{\widetilde{m}[X]}(Y-X)^{2}+\tilde{\tau}\left|\nabla_{m[X]} Y\right|^{2} \mathrm{~d} a \\
& =\widetilde{\mathbf{M}}[X]\left(\bar{Y}-\mathbf{E} \bar{X}-\bar{X}^{\text {ext }}\right) \cdot\left(\bar{Y}-\mathbf{E} \bar{X}-\bar{X}^{\text {ext }}\right)+\tilde{\tau} \widetilde{\mathbf{L}}[X] \bar{Y} \cdot \bar{Y},
\end{aligned}
$$

where $\widetilde{\mathbf{M}}[X]$ and $\widetilde{\mathbf{L}}[X]$ are the block mass and stiffness matrices on $\boldsymbol{V}(\widetilde{m}[X])$. Hence, now the nodal vector $\bar{Y}[X]$ is still in $\mathbb{R}^{m|I|}$ and solves

$$
(\widetilde{\mathbf{M}}[X]+\tilde{\tau} \widetilde{\mathbf{L}}[X]) \bar{Y}[X]=\widetilde{\mathbf{M}}[X]\left(\mathbf{E} \bar{X}-\bar{X}^{\text {ext }}\right) .
$$

\subsection{Anisotropic Willmore flow of closed surfaces}

The spatial discretization of the anisotropic Willmore flow of hyper-surfaces is based on the discrete functional

$$
\begin{aligned}
E_{\gamma}[X, Y] & :=\int_{m[X]}(Y-X)^{2} \mathrm{~d} a+2 \tilde{\tau} \int_{m[Y]} \gamma(N[Y]) \mathrm{d} a \\
& =\mathbf{M}[X](\bar{Y}-\bar{X}) \cdot(\bar{Y}-\bar{X})+2 \tilde{\tau} A_{\gamma}[Y]
\end{aligned}
$$


with $A_{\gamma}[Y]=\int_{m[Y]} \gamma(N[Y]) \mathrm{d} a$ being the anisotropic area functional. Here, $\gamma(N[Y])$ is piecewise constant on $m[Y]$ and thus can be integrated exactly. Furthermore, the functional $W\left[X^{k}, \cdot, \cdot\right]$ remains the same as in the isotropic case. Now, for fixed $X$ the discrete Euler Lagrange equation with respect to $Y$ is obviously nonlinear and we obtain that $\bar{Y}$ solves

$$
0=\mathbf{M}[X](\bar{Y}-\bar{X})+\tilde{\tau} \operatorname{grad} A_{\gamma}[Y]
$$

where the gradient is assembled via the evaluating of

$$
\partial_{Y} A_{\gamma}[Y](\Theta)=\int_{m_{[Y]}} \gamma(N[Y]) \nabla_{\mathfrak{m}[Y]} Y: \nabla_{\mathfrak{m}[Y]} \Theta \mathrm{d} a-\int_{m_{[Y]}} \sum_{l=1}^{d+1} \gamma_{z}(N[Y]) \cdot \nabla_{\mathfrak{m}[Y]} \Theta_{l} N_{l}[Y] \mathrm{d} a
$$

for all basis functions $\Theta=\Phi_{i} e_{j}$. Here, $N[Y]$ is the piecewise constant normal field on $m[Y]$ and the first integral on the right hand side represents the variation of the anisotropic area functional with respect to the integration domain taking into account the linearization argument from Appendix A. Furthermore, the second integral reflects the variation of the integrant making use of the observation

$$
\left.\frac{\mathrm{d}}{\mathrm{d} \epsilon} \gamma(N[Y+\epsilon \Theta])\right|_{\epsilon=0}=-\sum_{l=1}^{d+1} \gamma_{z}(N[Y]) \cdot \nabla_{m[Y]} \Theta_{l} N_{l}[Y] .
$$

For a given $X$, equation (3.4) can be solved by a Newton method.

\section{Optimization algorithm for the time steps}

In Section 2 we have derived a variational time discretization for different Willmore flow models leading to a constrained optimization problem, where the constraint is associated with the approximation of the isotropic or anisotropic mean curvature via the time discrete speed of a corresponding curvature motion model. Through spatial discretization we formulated in Section 3 a finite dimensional constrained optimization problem to be solved in each time step of Willmore flow. Here, we now use classical duality techniques from optimization to solve the resulting time step problem efficiently. For a general overview on these techniques we refer to Nocedal \& Wright [39] or $[4,28,30]$.

\subsection{Duality approach and gradient descent}

To simplify the exposition we first restrict ourselves to the isotropic Willmore flow model for closed surfaces. Afterwards, we will generalize this approach for surfaces with boundaries and anisotropic Willmore flow. Slightly rephrasing (3.1) and (3.2) we aim at minimizing the functional

$$
\widehat{W}[X]=W\left[X^{k}, X, Y[X]\right]
$$

with $Y[X]$ being a solution of the inner minimization problem (3.2). By $\partial_{X}$ we denote the differentiation with respect to vector $\bar{X}$ of grid nodes. A straightforward differentiation leads to

$$
\partial_{X} \widehat{W}[X](\Theta)=\partial_{X} W\left[X^{k}, X, Y[X]\right](\Theta)+\left(\partial_{Y} W\right)\left[X^{k}, X, Y[X]\right]\left(\partial_{X} Y[X](\Theta)\right) .
$$


Hence, computing the full gradient of $\widehat{W}$ in this way requires the evaluation of $\partial_{X} Y[X]\left(\Phi_{i} e_{j}\right)$ for every finite element basis function $\Phi_{i}$ and any canonical basis vector $e_{j}$ in $\mathbb{R}^{m}$. To derive a computational efficient representation of the gradient as an essential ingredient of any descent algorithm, we apply the following duality argument. From the optimality of $Y[X]$ in the inner problem, we deduce the equation $0=\partial_{Y} E[X, Y[X]](\Psi)$ for any test function $\Psi \in V(\mathfrak{m}[X])^{m}$. Differentiating with respect to $X$ we obtain

$$
\begin{aligned}
0 & =\partial_{X}\left(\partial_{Y} E[X, Y[X]](\Psi)\right)(\Theta) \\
& =\partial_{X} \partial_{Y} E[X, Y](\Psi, \Theta)+\partial_{Y}^{2} E[X, Y[X]]\left(\Psi, \partial_{X} Y[X](\Theta)\right)
\end{aligned}
$$

Now, one defines $P \in \mathcal{V}\left(\mathfrak{m}\left[X^{k}\right]\right)^{m}$ as the solution of the dual problem

$$
\partial_{Y}^{2} E[X, Y[X]](P, \Psi)=\partial_{Y} W\left[X^{k}, X, Y[X]\right](\Psi)
$$

for all test functions $\Psi \in \mathcal{V}\left(m\left[X^{k}\right]\right)^{m}$. Choosing $\Psi=\partial_{X} Y[X](\Theta)$ in (4.2) and $\Psi=P$ in (4.1) yields

$$
\left(\partial_{Y} W\right)\left[X^{k}, X, Y[X]\right]\left(\partial_{X} Y[X](\Theta)\right)=-\partial_{X} \partial_{Y} E[X, Y](P, \Theta) .
$$

Thus, we can finally rewrite the variation of $\widehat{W}$ as

$$
\partial_{X} \widehat{W}[X](\Theta)=\partial_{X} W\left[X^{k}, X, Y[X]\right](\Theta)-\partial_{X} \partial_{Y} E[X, Y](P, \Theta)
$$

In the case of our basic Willmore flow model (3.1) and (3.2) the solution $P$ of the dual problem (4.2) solves the linear system of equations

$$
(\mathbf{M}[X]+\tilde{\tau} \mathbf{L}[X]) \bar{P}=\frac{\tau}{\tilde{\tau}^{2}} \mathbf{M}[X](\bar{Y}-\bar{X}) .
$$

Here $\mathbf{M}[X]$ and $\mathbf{L}[X]$ are $m \times m$ block diagonal matrices, those diagonal blocks are the usual $|I| \times|I|$ mass matrices $M[X]$ and stiffness matrices $L[X]$, respectively. Furthermore, the terms on the right hand side of (4.3) are evaluated as follows

$$
\begin{aligned}
\left(\partial_{X} W\right)\left[X^{k}, X, Y\right](\Theta)= & 2 \mathbf{M}\left[X^{k}\right]\left(\bar{X}-\bar{X}^{k}\right) \cdot \bar{\Theta}+2 \frac{\tau}{\tilde{\tau}^{2}} \mathbf{M}[X](\bar{X}-\bar{Y}) \cdot \bar{\Theta} \\
& +\frac{\tau}{\tilde{\tau}^{2}}\left(\partial_{X} \mathbf{M}[X](\Theta)\right)(\bar{Y}-\bar{X}) \cdot(\bar{Y}-\bar{X}) \\
\partial_{X} \partial_{Y} E[X, Y](P, \Theta)=2 & \left.2 \partial_{X} \mathbf{M}[X](\Theta)\right)(\bar{Y}-\bar{X}) \cdot \bar{P}-2 \mathbf{M}[X] \bar{\Theta} \cdot \bar{P} \\
& +2 \tilde{\tau}\left(\partial_{X} \mathbf{L}[X](\Theta)\right) \bar{Y} \cdot \bar{P}
\end{aligned}
$$

Hence, we need to compute the variation $\partial_{X} \mathbf{M}[X](\Theta)$ and $\partial_{X} \mathbf{L}[X](\Theta)$ of the block matrices $\mathbf{M}$ and $\mathbf{L}$, respectively, where $\Theta$ represents a variation of the simplicial mesh. The variation of these block matrices composes of diagonal blocks of the variations of the corresponding matrices from the scalar case defined as $\partial_{X} M[X](\Theta)=\left.\frac{\mathrm{d}}{\mathrm{d} \epsilon} M[X+\epsilon \Theta]\right|_{\epsilon=0}$ and $\partial_{X} L[X](\Theta)=\left.\frac{\mathrm{d}}{\mathrm{d} \epsilon} L[X+\epsilon \Theta]\right|_{\epsilon=0}$. They are computed using a standard finite element assembly. We refer to Appendix B for the actual differentiation of the corresponding local matrix entries. With these ingredients at hand we can finally compute the descent direction in $\mathbb{R}^{m|I|}$ of the energy $\widehat{W}$ for a given simplicit mesh $m[X]$ described by the nodal vector $\bar{X}$ to obtain

$$
\operatorname{grad}_{X} \widehat{W}[X]=\left(\partial_{X} \widehat{W}[X]\left(\Phi_{r} e_{s}\right)\right)_{r \in I, s=1, \cdots, m},
$$


where $e_{s}$ denotes the $s$ th coordinate direction in $\mathbb{R}^{m}$.

With this gradient evaluation at hand we can already apply a gradient descent scheme with the Amijo step size control, choosing as initial data the nodal vector $\bar{X}^{k}$ from the previous time step.

In the case of Willmore flow with boundary conditions, we investigate the functional $\widehat{W}^{\partial}[X]:=$ $W^{\partial}\left[X^{k}, X, Y[X]\right]$. Using the same duality argument $P$ solves

$$
(\mathbf{M}[X]+\tilde{\tau} \mathbf{L}[X]) \bar{P}=\frac{\tau}{\tilde{\tau}^{2}} \mathbf{M}[X]\left(\bar{Y}-\mathbf{E} \bar{X}-\bar{X}^{\text {ext }}\right),
$$

or in the alternative approach

$$
(\widetilde{\mathbf{M}}[X]+\tilde{\tau} \widetilde{\mathbf{L}}[X]) \bar{P}=\frac{\tau}{\tilde{\tau}^{2}} \mathbf{M}[X]\left(\bar{Y}-\mathbf{E} \bar{X}-\bar{X}^{e x t}\right) .
$$

Furthermore, in this case given $\partial_{X} \widehat{W}^{\partial}[X](\Theta)=\partial_{X} W^{\partial}\left[X^{k}, X, Y[X]\right](\Theta)-\partial_{X} \partial_{Y} E^{\partial}[X, Y](P, \Theta)$ the gradient components on the right hand side are evaluated as follows

$$
\begin{array}{rl}
\partial_{X} W^{\partial}\left[X^{k}, X, Y\right](\Theta)=2 & \mathbf{M}\left[X^{k}\right]\left(\mathbf{E} \bar{X}-\bar{X}^{k}\right) \cdot \mathbf{E} \bar{\Theta}+2 \frac{\tau}{\tilde{\tau}^{2}} \mathbf{M}[X]\left(\mathbf{E} \bar{X}+\bar{X}^{e x t}-\bar{Y}\right) \cdot \mathbf{E} \bar{\Theta} \\
+ & \frac{\tau}{\tilde{\tau}^{2}}\left(\partial_{X} \mathbf{M}[X](\Theta)\right)\left(\bar{Y}-\mathbf{E} \bar{X}-\bar{X}^{\text {ext }}\right) \cdot\left(\bar{Y}-\mathbf{E} \bar{X}-\bar{X}^{\text {ext }}\right), \\
\partial_{X} \partial_{Y} E^{\partial}[X, Y](P, \Theta)=2 & \left(\partial_{X} \mathbf{M}[X](\Theta)\right)\left(\bar{Y}-\mathbf{E} \bar{X}-\bar{X}^{\text {ext }}\right) \cdot \bar{P}-2 \mathbf{M}[X] \mathbf{E} \bar{\Theta} \cdot \bar{P} \\
+ & 2 \tilde{\tau}\left(\partial_{X} \mathbf{L}[X](\Theta)\right) \bar{Y} \cdot \bar{P}
\end{array}
$$

whereas in the alternative approach the mass matrix $\mathbf{M}[X]$ and the stiffness matrix $\mathbf{L}[X]$ are replaced by $\widetilde{\mathbf{M}}[X]$ and $\widetilde{\mathbf{L}}[X]$, respectively.

Let us finally investigate the fully nonlinear, anisotropic model. Now, the dual solution $P$ solves

$$
\left(\mathbf{M}[x]+\tilde{\tau} \operatorname{Hess} A_{\gamma}(Y)\right) \bar{P}=\frac{\tau}{\tilde{\tau}} \mathbf{M}[X](\bar{Y}-\bar{X}) .
$$

Here, as in the gradient case, the Hessian is assembled via evaluation of $\partial_{Y}^{2} A_{\gamma}[Y]\left(\Phi_{i} e_{j}, \Phi_{k} e_{l}\right)$ for all vector valued basis functions $\Phi_{i} e_{j}$ and $\Phi_{k} e_{l}$, respectively. In the Appendix C we give formulas for the computation of the local entries Hess $A_{\gamma}[Y]$ on single elements in the case of polygonal curves $(d=1$ and $m=2)$. Finally, for the second term in the gradient formula $\partial_{X} \widehat{W}^{\partial}[X](\Theta)=$ $\partial_{X} W^{\partial}\left[X^{k}, X, Y[X]\right](\Theta)-\partial_{X} \partial_{Y} E_{\gamma}[X, Y](P, \Theta)$ we obtain

$$
\partial_{X} \partial_{Y} E_{\gamma}[X, Y](P, \Theta)=2\left(\partial_{X} \mathbf{M}[X](\Theta)\right)(\bar{Y}-\bar{X}) \cdot \bar{P}-2 \mathbf{M}[X] \bar{\Theta} \cdot \bar{P} .
$$

\subsection{Lagrangian and SQP method}

A more efficient strategy to solve the constrained optimization problem in each time step is to apply a Newton method for the corresponding Lagrangian, which can also be phrased as a sequential quadratic programming (SQP) approach. For an introduction to the SQP method and the basic convergence theory we refer to Nocedal \& Wright [39]. In our context we consider the following Lagrangian function for problem (3.1), (3.2)

$$
L[\bar{X}, \bar{Y}, \bar{P}]=W\left[X^{k}, X, Y\right]-\partial_{Y} E[X, Y](P)
$$


for the now independent unknowns $\bar{X}, \bar{Y} \in \mathbb{R}^{m|I|}$ and the Lagrange multiplier $\bar{P} \in \mathbb{R}^{m|I|}$. Here, with a slight misuse of notation, we either use a finite element function notation with $Z$ in $V\left(m\left[X^{k}\right]\right)^{m}$ and $(m[X])^{m}$, respectively, or the corresponding nodal vector notation with $\bar{Z} \in \mathbb{R}^{m|I|}$. Now, we ask for critical points $(\bar{X}, \bar{Y}, \bar{P})$ of $L$. Indeed, $0=\partial_{\bar{P}} L[\bar{X}, \bar{Y}, \bar{P}](\bar{\Theta})=$ $\partial_{Y} E[X, Y](\bar{\Theta})$ is the Euler Lagrange equation of the inner minimization problem with respect to $\bar{Y}$ for given $X$ and $0=\partial_{\bar{Y}} L[\bar{X}, \bar{Y}, \bar{P}](\bar{\Theta})=\partial_{Y} W\left[X^{k}, X, Y\right](\Theta)-\partial_{Y}^{2} E[X, Y](P, \Theta)$ is the defining equation for the dual solution $P$ given $Y$ as the solution of the above Euler Lagrange equation. Finally, $0=\partial_{\bar{X}} L[\bar{X}, \bar{Y}, \bar{P}](\bar{\Theta})=\partial_{X} W\left(X^{k}, X, Y\right)(\Theta)-\partial_{X} \partial_{Y} E[X, Y](P, \Theta)=\partial_{X} \widehat{W}(X)$ coincides with the Euler Lagrange equation for the actual constraint optimization problem. The Hessian of $L$, which is required to implement a Newton scheme, is given (in abbreviated form) by

$$
\text { Hess } L=\left(\begin{array}{ccc}
\partial_{X}^{2} W-\partial_{X}^{2} \partial_{Y} E(P) & \partial_{X} \partial_{Y} W-\partial_{X} \partial_{Y}^{2} E(P) & -\partial_{X} \partial_{Y} E \\
\partial_{X} \partial_{Y} W-\partial_{X} \partial_{Y}^{2} E(P) & \partial_{Y}^{2} W-\partial_{Y}^{3} E(P) & -\partial_{Y}^{2} E \\
-\partial_{X} \partial_{Y} E & -\partial_{Y}^{2} E & 0
\end{array}\right)
$$

By a straightforward Lagrangian multiplier argument a Newton step coincides with the solution of the quadratic program

$$
\left(\frac{1}{2} \operatorname{Hess}_{(\bar{X}, \bar{Y})} L\left[\bar{X}^{k, l}, \bar{Y}^{k, l}, \bar{P}^{k, l}\right](\Delta \bar{X}, \Delta \bar{Y})+\operatorname{grad}_{(\bar{X}, \bar{Y})} W\left[\bar{X}^{k, l}, \bar{Y}^{k, l}\right]\right) \cdot(\Delta \bar{X}, \Delta \bar{Y}) \rightarrow \min
$$

subject to the linearized constraint $\partial_{\bar{Y}} E\left[\bar{X}^{k, l}, \bar{Y}^{k, l}\right]+\operatorname{grad}_{(\bar{X}, \bar{Y})} \partial_{\bar{Y}} E\left[\bar{X}^{k, l}, \bar{Y}^{k, l}\right] \cdot(\Delta \bar{X}, \Delta \bar{Y})=0$, where the index $l$ indicates the $l$ th Newton step with $\bar{X}^{k, l+1}=\bar{X}^{k, l}+\Delta \bar{X}, \bar{Y}^{k, l+1}=\bar{Y}^{k, l}+\Delta \bar{Y}$ and $\bar{P}^{k, l+1}$ appears as the Lagrangian multiplier with respect to the linear constraint.

For the Willmore flow of closed surfaces leading to the optimization problem (3.1), (3.2) the different terms in Hess $L$ are evaluated as follows:

$$
\begin{aligned}
\partial_{X}^{2} W(\Theta, \Psi)= & 2\left(\mathbf{M}\left[X^{k}\right]+\frac{\tau}{\tilde{\tau}^{2}} \mathbf{M}[X]\right) \bar{\Psi} \cdot \bar{\Theta}+\frac{\tau}{\tilde{\tau}^{2}}\left(\partial_{X}^{2} \mathbf{M}[X](\Theta, \Psi)(\bar{Y}-\bar{X}) \cdot(\bar{Y}-\bar{X})\right. \\
& \left.+2 \partial_{X} \mathbf{M}[X](\Theta)(\bar{X}-\bar{Y}) \cdot \bar{\Psi}+2 \partial_{X} \mathbf{M}[X](\Psi)(\bar{X}-\bar{Y}) \cdot \bar{\Theta}\right), \\
\partial_{X} \partial_{Y} W(\Theta, \Psi)= & 2 \frac{\tau}{\tilde{\tau}^{2}}\left(\partial_{X} \mathbf{M}[X](\Psi)(\bar{Y}-\bar{X}) \cdot \bar{\Theta}-\mathbf{M}[X] \bar{\Theta} \cdot \bar{\Psi}\right) \\
\partial_{Y}^{2} W(\Theta, \Psi)= & 2 \frac{\tau}{\tilde{\tau}^{2}} \mathbf{M}[X] \bar{\Theta} \cdot \bar{\Psi} \\
\partial_{X}^{2} \partial_{Y} E(\Theta, \Psi, \Xi)= & 2\left(\partial_{X}^{2} \mathbf{M}[X](\Psi, \Xi)(\bar{Y}-\bar{X}) \cdot \bar{\Theta}-\partial_{X} \mathbf{M}[X](\Psi) \bar{\Theta} \cdot \bar{\Xi}\right. \\
\partial_{X} \partial_{Y}^{2} E(\Theta, \Psi, \Xi)= & 2\left(\partial_{X} \mathbf{M}[X](\Xi)+\tilde{\tau} \partial_{X} \mathbf{L}[X](\Xi)\right) \bar{\Theta} \cdot \bar{\Psi}, \\
\partial_{X} \partial_{Y} E(\Theta, \Psi)= & 2\left(\partial_{X} \mathbf{M}[X](\Psi)(\bar{Y}-\bar{X}) \cdot \bar{\Theta}-\mathbf{M}[X] \bar{\Psi} \cdot \bar{\Theta}+\tilde{\tau} \partial_{X} \mathbf{L}[X](\Psi) \bar{Y} \cdot \bar{\Theta}\right), \\
\partial_{Y}^{3} E= & 0, \\
\partial_{Y}^{2} E(\Theta, \Psi)= & 2(\mathbf{M}[X]+\tilde{\tau} \mathbf{L}[X]) \bar{\Theta} \cdot \bar{\Psi},
\end{aligned}
$$

where $\partial_{X} \partial_{Y} E$ has already been given in (4.5). The different sub blocks of the block structured Hessian Hess $L$ are again assembled in the usual way from local contribution on single elements of the polygonal mesh. Detailed formulas for the local derivatives are given in Appendix B.

The adaptation in the case of Willmore flow with boundary conditions is obvious, whereas for anisotropic Willmore flow we obtain for the derivatives of the energy $E_{\gamma}$ from (3.3) appearing in 

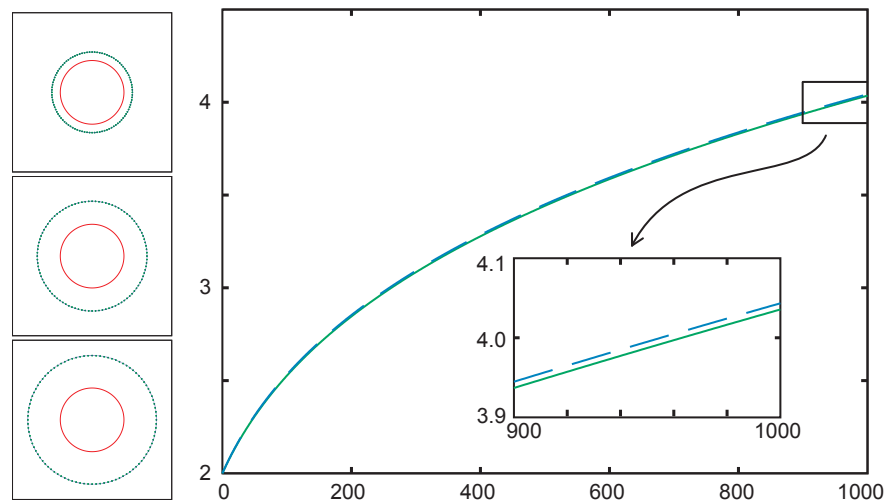

FIG. 2. The evolution of a circle under Willmore flow is displayed. On the left, the initial circle of radius $R(0)=2$ (red) and the discrete solution (green) are plotted at times $t=0.01,0.05,0.1$. The computation is based on a polygonal approximation with 200 vertices and $\tau=\tilde{\tau}=h$. Furthermore on the right, the evolution of the average radius (green) is compared with the radius of the exact solution (blue) over time.

Hess $L$

$$
\begin{aligned}
\partial_{X}^{2} \partial_{Y} E_{\gamma}(\Theta, \Psi, \Xi)= & 2\left(\partial_{X}^{2} \mathbf{M}[X](\Psi, \Xi)(\bar{Y}-\bar{X}) \cdot \bar{\Theta}\right. \\
& \left.-\partial_{X} \mathbf{M}[X](\Psi) \bar{\Xi} \cdot \bar{\Theta}-\partial_{X} \mathbf{M}[X](\Xi) \bar{\Psi} \cdot \bar{\Theta}\right), \\
\partial_{X} \partial_{Y}^{2} E_{\gamma}(\Theta, \Psi, \Xi)= & 2 \partial_{X} \mathbf{M}[X](\Xi) \bar{\Psi} \cdot \bar{\Theta}, \\
\partial_{X} \partial_{Y} E_{\gamma}(\Theta, \Psi)= & 2\left(\partial_{X} \mathbf{M}[X](\Psi)(\bar{Y}-\bar{X}) \cdot \bar{\Theta}-\mathbf{M}[X] \bar{\Psi} \cdot \bar{\Theta}\right), \\
\partial_{Y}^{3} E_{\gamma}(\Theta, \Psi, \Xi)= & 2 \tilde{\tau} \partial_{Y} \operatorname{Hess} A_{\gamma}[Y](\Xi) \bar{\Psi} \cdot \bar{\Theta}, \\
\partial_{Y}^{2} E_{\gamma}(\Theta, \Psi)= & 2\left(\mathbf{M}[X]+\tilde{\tau} \operatorname{Hess} A_{\gamma}[Y]\right) \bar{\Psi} \cdot \bar{\Theta} .
\end{aligned}
$$

For the (now necessary) third derivatives of the local anisotropic area functional $A_{\gamma}$ we refer to Appendix C.

\section{Numerical results}

We have applied our numerical algorithm for Willmore flow to the evolution of curves in $\mathbb{R}^{2}$ and in $\mathbb{R}^{3}$, and to two dimensional surfaces in $\mathbb{R}^{3}$. We study curve and surface blending problems and investigate the numerical solution of anisotropic Willmore of curves. A particular emphasis is on the robustness and stability of the proposed approach in particular for large time steps $\tau$ up to the order the spatial grid size $h$.

\subsection{Willmore flow of closed curves and surfaces}

At first, we numerical solve the Willmore flow problem for curves in 2D and simulate the evolution of circles, where the explicit solution has already been discussed in Section 2.1. The continuous and discrete evolution is compared in Fig. 2 and an experimental study of convergence in the $L^{2}$ norm is presented in Table 1 in agreement with the consistency error of our model $O\left(h^{2}+\tau+\tilde{\tau}\right)$. For 
TABLE 1. The $L^{2}$ error between the exact solution $R(t)$ for $t=0.01$ and the corresponding discrete radius function $R_{h}\left(X^{k}\right):=\left|X^{k}\right|$ with $\tau=\frac{t}{k}$ is computed on $m\left[X^{k}\right]$, where $\tau=\tilde{\tau}=h^{2}$ (left column) and $\tau=\tilde{\tau}=h$ (right column) for a varying grid size $h$ resulting form a initial polygon $m\left[X^{0}\right]$ with $2^{n}$ edges of equal length with $n=4,5,6,7,8$.

\begin{tabular}{ccc}
\hline$n$ & $L^{2}$ error $\left(\tau=\tilde{\tau}=h^{2}\right)$ & $L^{2}$ error $(\tau=\tilde{\tau}=h)$ \\
\hline 4 & 0.1255 & 0.1632 \\
5 & 0.0425 & 0.0697 \\
6 & 0.0098 & 0.0294 \\
7 & 0.0032 & 0.0137 \\
8 & 0.0006 & 0.0057 \\
\hline
\end{tabular}

the actual numerical solution of the discrete variational problem to be solved at each time step we have used the Newton scheme for the Lagrangian.

Furthermore, we investigate spheres in $\mathbb{R}^{3}$, which are known to be stationary solutions of Willmore flow. The Willmore energy is invariant with respect to Möbius transformation. These invariants might lead to degeneracies of the numerical grid in long time simulations as observed for the semi-implicit algorithm of Rusu [47]. On the same time-scale and with the same underlying time step size the nested time discretization turned out to be more robust as depicted in Fig. 3. Even for significantly larger times the new scheme remains stable.

Next, we consider an example already proposed by Dziuk and Deckelnick in [15], where a hypocycloid is considered as initial data. Here, the parametrization of the initial curve is given by $X_{0}(t)=\left(-\frac{5}{2} \cos (t)+4 \cos (5 t),-\frac{5}{2} \sin (t)+4 \sin (5 t), \delta \sin (3 t)\right)$. In $\mathbb{R}^{2}$ for $\delta=0$ the initial curve evolves to a fivefold covering of a circle (cf. Figure 4) since multiple coverings of a circle are stable stationary solutions in the co-dimension one case [44]. This is not true in the case of higher
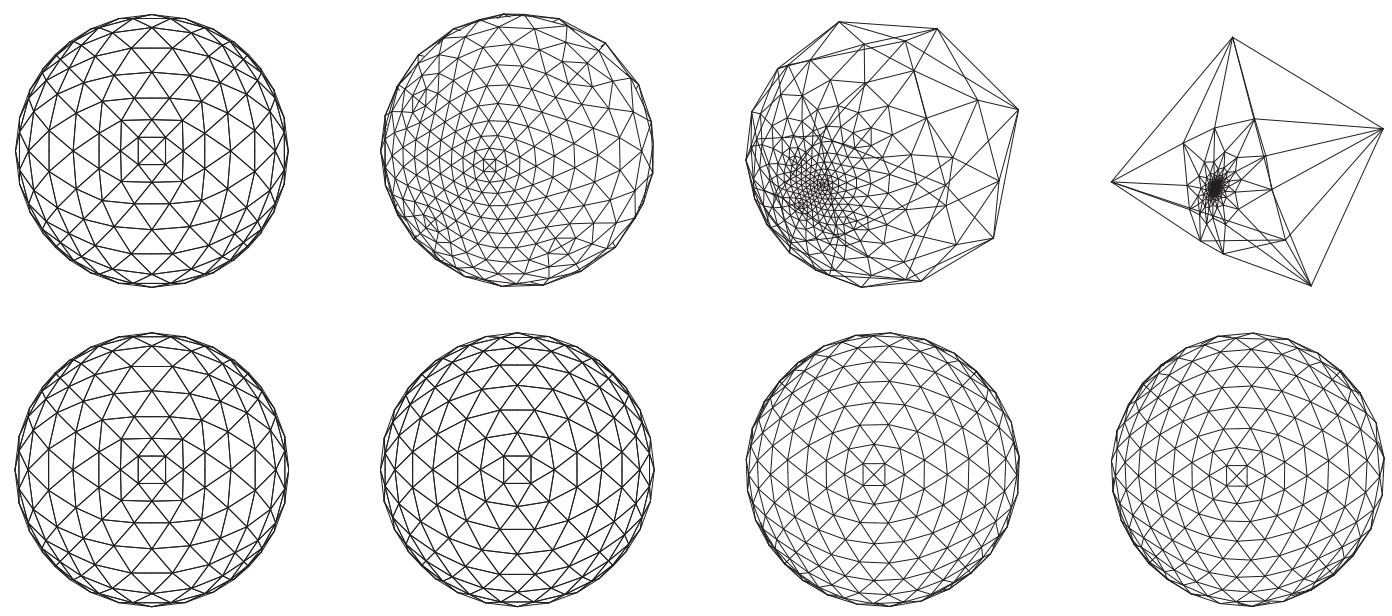

FIG. 3. Results of the semi-implicit scheme [47] (top row) are compared with the proposed algorithm using a Newton method (bottom row). The mesh size of the discrete spheres is $h=0.02$, and as time step size we consider $\tau=16 \cdot 10^{-8}$ in both methods ( $\tilde{\tau}=\tau$ in the our scheme). From left to right the triangulations are rendered at times $t=0.0, t=2381.9$, $t=2442,2, t=2472,4$. 

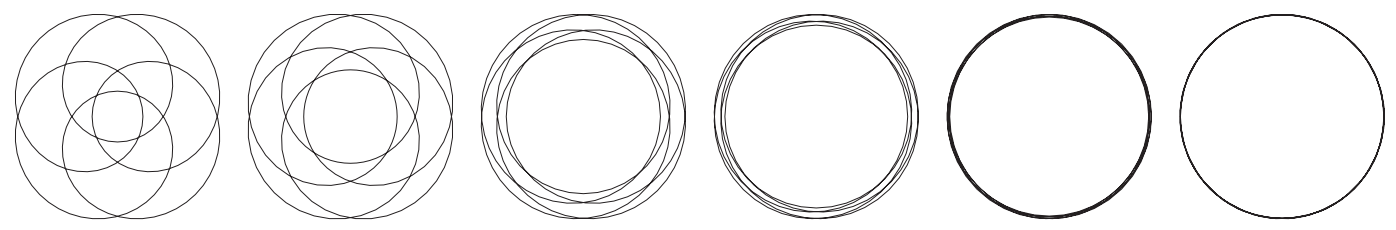

FIG. 4. The evolution of a planar hypocycloid towards a fivefold covering of a circle is shown at times $t=0.0, t=685.7$, $t=2987.4, t=4850.1, t=7965.8, t=10630.6$. The curves are graphically rescaled to have similar size. The computational parameters were $\lambda=0.025$, and $\tau=h=0.5493$ and a gradient descent method was used.
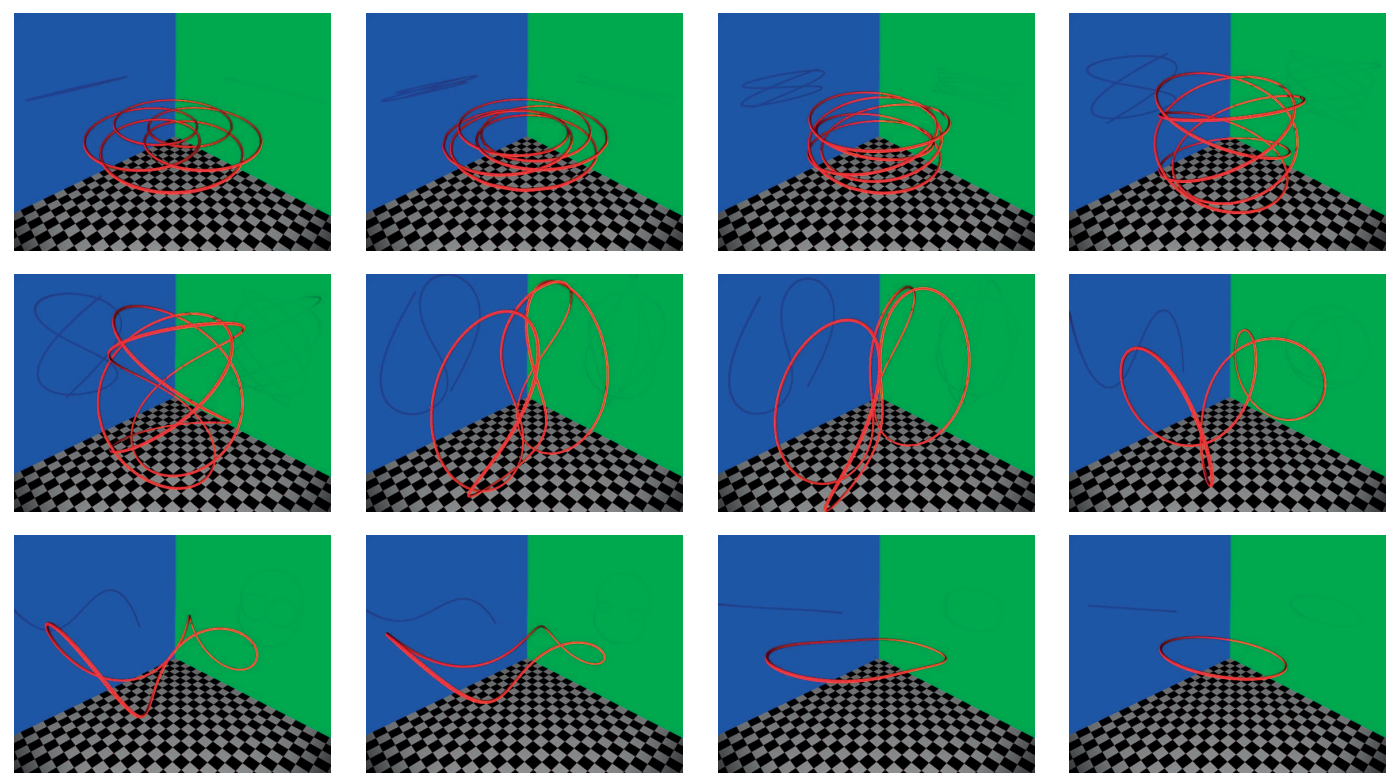

FIG. 5. The evolution of a vertically perturbed hypocycloid towards a circle under Willmore flow with $\delta=0.1, h=0.005$ at $t=0$, and $\tau=0.005$ is shown at times $t=0.0, t=1348.9, t=4467.1, t=5511.4, t=6555.7, t=7406.6$, $t=8257.2, t=9108.4, t=9297.0, t=9361.3, t=9426.8, t=9489.1$.

co-dimension for $m \geqslant 3$. If we start with an initial curve slightly perturbed in vertical direction, we have chosen $\delta=0.1$, the curve begins to unfold and evolves to a single circle (cf. Figure 5). Here, we use a gradient descent method to solve the optimization problem for each time step.

Furthermore, we depict in Figure 6 the evolution of a coarse polygonal approximation of a torus towards the Clifford torus $m=\left\{x \in \mathbb{R}^{3} \mid\left(1-\sqrt{x_{1}^{2}+x_{2}^{2}}\right)^{2}+x_{3}^{2}=\frac{1}{2}\right\}$. In Figure 7 we compare the discrete evolution at a fixed time for different choices of the time step $\tau(\tilde{\tau}=\tau)$.

\subsection{Curve and surface blending}

Figure 8 shows different solutions of a curve blending problem. The Willmore energy for a circular segment with an opening angle $\alpha$ and radius $r$ is given by $\frac{\alpha}{r}$. Thus, for two given curve segments which have to be blended we can continue these segments by straight line segments and connect them by a circular arc. As the length of the straight line segments tends to infinity the Willmore 

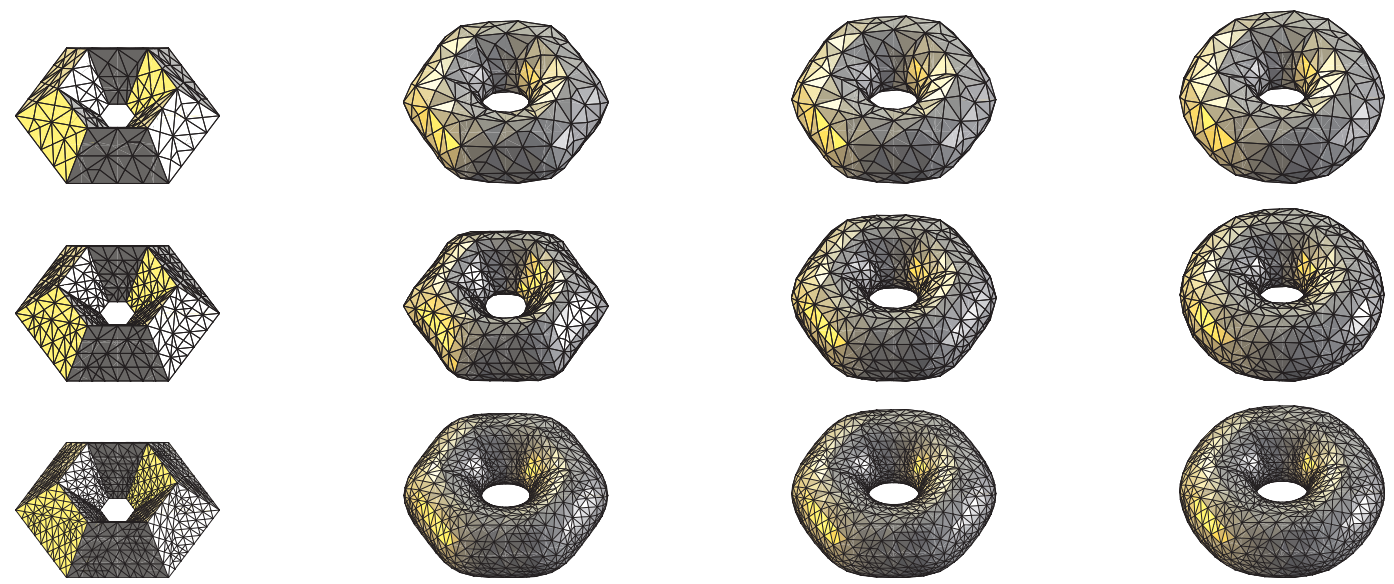

FIG. 6. Different Willmore flow time steps are depicted from the evolution of a coarse polygonal torus model towards the Clifford torus. The Newton scheme for the Lagrangian is applied, where $h=0.0977$ (1st row), $h=0.0745$ (2nd row), and $h=0.0089$ (3rd row), respectively. From left to right the surfaces are rendered at times $t=0.0, t=0.09, t=0.15$, and $t=0.97$.
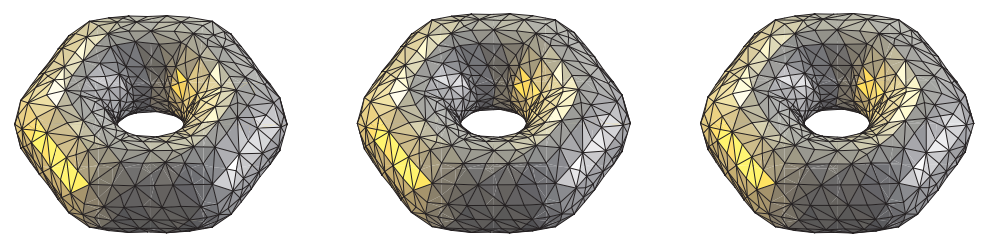

FIG.7. For the Willmore flow evolution in Figure 6) we render the triangulated discrete surfaces at time $t=0.3735$ resulting from computations with different time step sizes $\tau=h^{4}, \tau=h^{2}$, and $\tau=h$, where the initial grid size is $h=0.0745$.
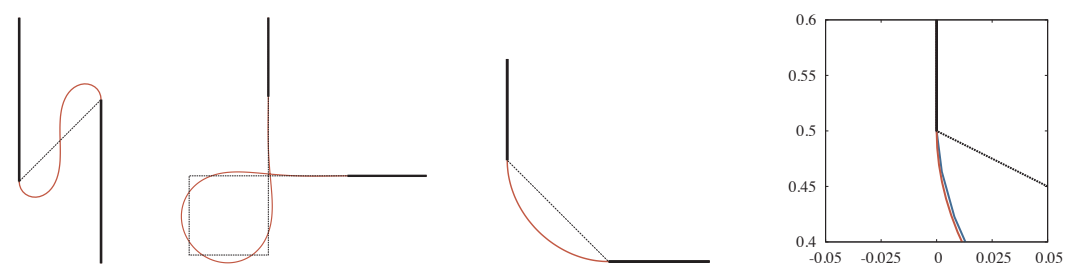

FIG. 8. Discrete stationary solutions of different curve blending problem are shown. Here, $\tau=h=0.01386, \tilde{\tau}=$ $1.386 \cdot 10^{-5}$, and $\lambda=1.0$. The initial curve is plotted as a dotted line. On the right we compare the scheme with prescribed co-normal $N^{c o}$ (red) and the alternative approach with an additional ring of triangles (blue) in an enlargement of the previous plot.

energy of the whole blending construction tends to zero. To avoid this modeling artifact we consider a slight generalization of the above Willmore flow model. We add $\lambda a[x]$ to the Willmore energy, where $\lambda$ is a fixed constant and $a[x]$ denotes the length of the curve. Here, $\lambda$ can be regarded as a Lagrangian multiplier with respect to a length constraint. Hence, for proper choices of $\lambda$ the generalized model avoids expansion. If $X$ represents a discrete closed curve as above, we obtain for 

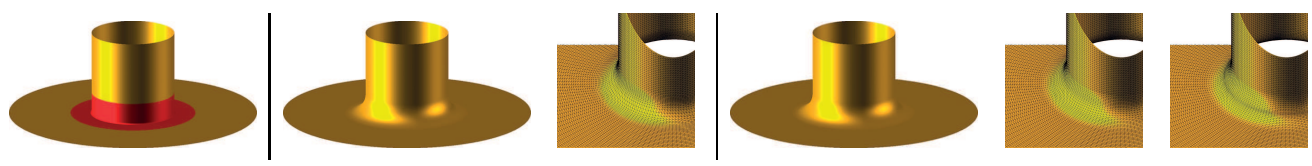

FIG. 9. For the solution of a surface blending problem with a blending region marked in red (left) we compare the semiimplicit scheme from [13] (middle block) and the new method using a SQP approach (right block) with a blow-up zoomed view onto the corresponding triangulation. Here, the (almost stationary) solution is rendered at time $t=1.4$ and the computational parameters are $h=\tau=0.14, \tilde{\tau}=0.002$ (first blow-up in on the right) and $\tau=\tilde{\tau}=0.0004$ (second blow-up on the right)
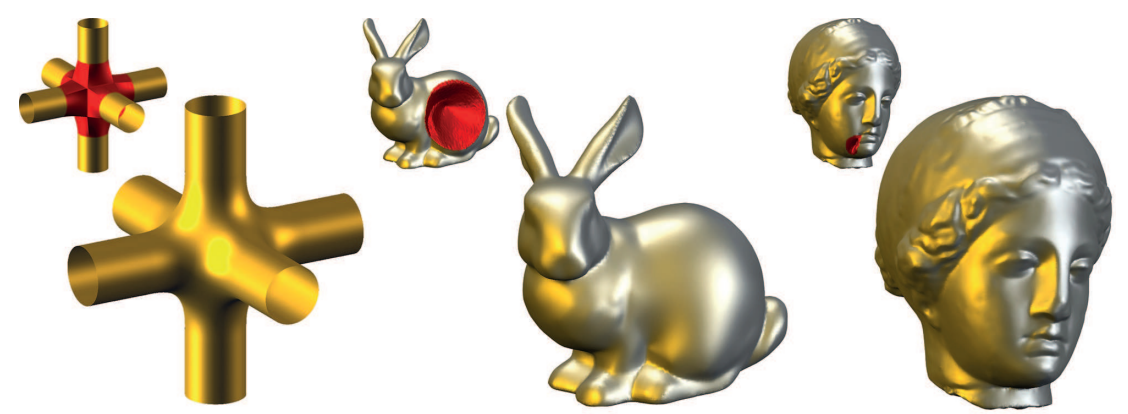

FIG. 10. Results of the second variant of the new method for Willmore flow are shown for a higher genus blending problem and two different surface restoration applications using the SQP approach.

the discrete length functional $A[X]=\sum_{i \in I} Q_{i}$. Furthermore, its gradient vector in $\mathbb{R}^{m|I|}$ is given by $\overline{\operatorname{grad}_{X} A[X]}=L[X] \bar{X}$. Figure 9 shows a blending surface generated by the proposed method and a comparison with the restoration method by Clarenz et al. [13] based on the semi-implicit discretization of Willmore flow.

In curve and surface restoration the two variants of our approach give very similar results and differ only slightly in the corresponding parameterization. The second variant appears to more natural for surface restoration with a given outer triangular mesh, whereas the first variation is more suitable for blending problems with explicitly given co-normal.

Finally, in Figure 10 we address further blending and surface restoration problems using the second variant of our method. For a comparison with the semi-implicit approach and a discrete geometry approach we refer to Clarenz et al. [13] and Bobenko \& Schröder [7], respectively.

\subsection{Simulation of anisotropic Willmore flow of curves}

We have implemented our numerical algorithm for the anisotropic Willmore flow of polygonal curves in $\mathbb{R}^{2}$. Here, we present results for three different types of Wulff shapes and corresponding anisotropies $\gamma_{0}(z)=|z|, \gamma_{1}(z)=\sqrt{z_{1}^{2}+4 z_{2}^{2}}$, and $\gamma_{2}(z)=\sum_{l=1}^{2} \sqrt{10^{-7}|z|^{2}+z_{l}^{2}}$ for $z=$ $\left(z_{1}, z_{2}\right) \in \mathbb{R}^{2}$. In addition, we take into account a length energy as in Section 5.2. The results underline that time steps up to the order the spatial grid size $h$ are feasible also in the anisotropic case. Figure 11 shows the evolution of a bunny shaped model towards a circle, an ellipse and a square with slightly rounded corners. We used the Newton method for the Lagrangian to solve the optimization problem at each time step. 

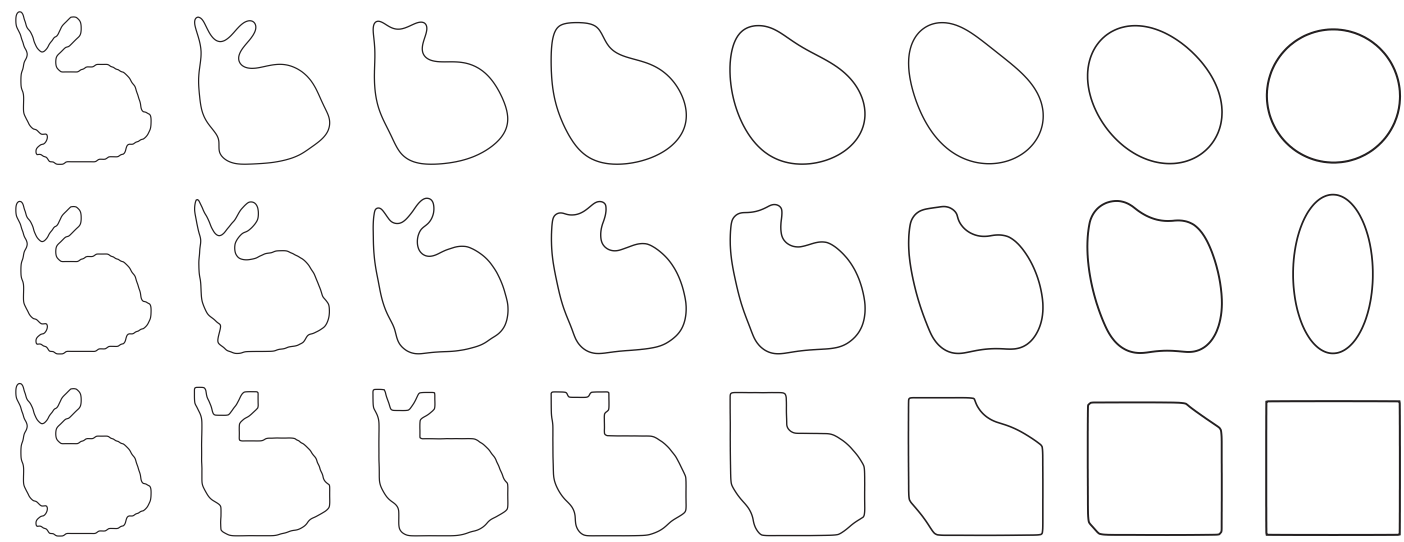

FIG. 11. Different time steps of the evolution of a bunny shape with 436 nodes towards a circle (first row), an ellipse (second row) In all three cases we have chosen $\tau=h=0.00865$ ( $h$ being the initial grid size), $\lambda=0.025$, and $\tilde{\tau}=7.5 \cdot 10^{-5}$.

\section{Appendix}

\section{A. Linearization of surface area}

Here, we show that for two $d$-dimensional surfaces $m[x]$ and $m[y]$ in $\mathbb{R}^{m}$ with smooth parametrizations $x$ and $y$ which are close in $C^{1}$, the approximation result (2.1) holds. To see this, let us consider $x$ and $y$ as two charts defined on a parameter domain $\omega \subset \mathbb{R}^{d}$ of $m[x]$ and $m[y]$, respectively. Then, $\int_{y(\omega)} \mathrm{d} a=\int_{\omega} \sqrt{\operatorname{det}\left(D y^{T} D y\right)} \mathrm{d} \xi$. Taking into account that $\left.\frac{\mathrm{d}}{\mathrm{d} s} \sqrt{d(1+s)}\right|_{s=0}=\frac{1}{2} \sqrt{d}$ and using Taylor expansion we obtain

$$
\begin{aligned}
\sqrt{\operatorname{det}\left(D y^{T} D y\right)}= & \sqrt{\operatorname{det}\left(D x^{T} D x\right) \operatorname{det}\left(1 \mathrm{I}+\left(D x^{T} D x\right)^{-1}\left(D y^{T} D y-D x^{T} D x\right)\right)} \\
= & \sqrt{\operatorname{det}\left(D x^{T} D x\right)}\left(1+\frac{\operatorname{tr}\left(\left(D x^{T} D x\right)^{-1}\left(D y^{T} D y-D x^{T} D x\right)\right)}{2}\right) \\
& \quad+O\left(\|y-x\|_{C^{1}(\omega)}^{2}\right) \\
= & \sqrt{\operatorname{det}\left(D x^{T} D x\right)}\left(1-\frac{d}{2}+\frac{1}{2} \operatorname{tr}\left(D y\left(D x^{T} D x\right)^{-1} D y^{T}\right)\right)+O\left(\|y-x\|_{C^{1}(\omega)}^{2}\right),
\end{aligned}
$$

where $\|f\|_{C^{1}(\omega)}$ denotes the $C^{1}$ norm of $f$ on the parameter domain $\omega$. Hence, we get

$$
\begin{aligned}
\int_{\omega} \sqrt{\operatorname{det}\left(D y^{T} D y\right)} \mathrm{d} \xi=( & \left.1-\frac{d}{2}\right) \int_{\omega} \sqrt{\operatorname{det}\left(D x^{T} D x\right)} \mathrm{d} \xi \\
& +\frac{1}{2} \int_{\omega} \operatorname{tr}\left(D y\left(D x^{T} D x\right)^{-1} D y^{T}\right) \sqrt{\operatorname{det}\left(D x^{T} D x\right)} \mathrm{d} \xi \\
& +O\left(\|y-x\|_{C^{1}(\omega)}^{2}\right),
\end{aligned}
$$

which together with the observation $\left|\nabla_{m[x]} y\right|^{2}=\operatorname{tr}\left(D y\left(D x^{T} D x\right)^{-1} D y^{T}\right)$ finally proves our claim. 


\section{B. Local finite element matrices and their derivatives}

Here, we derive formulas for the derivatives of the local mass and stiffness matrices. For simplicity we assume that we deal only with a single $d$ dimensional, non degenerate simplex $T$ with vertices $X_{0}, \cdots, X_{d}$ in $\mathbb{R}^{m}$. Then, the mass and stiffness matrix are given by

$$
M[X]=\sqrt{\operatorname{det} G[X]} \hat{M}, \quad L[X]=\frac{1}{d !} \sqrt{\operatorname{det} G[X]}\left(G[x]^{-1} \hat{\nabla} \hat{\Phi}_{i} \cdot \hat{\nabla} \hat{\Phi}_{j}\right)_{i, j=0, \ldots, d} .
$$

Here, $\hat{M}$ is the usual mass matrix on the reference simplex $\hat{T}$ in $\mathbb{R}^{d}$ with vertices 0 and $e_{1}, \cdots, e_{d}$. Furthermore, $\hat{\Phi}_{k}(k=0, \ldots, d)$ are the corresponding basis function on $\hat{T}$ and the gradient of $\hat{\Phi}_{k}$ is given by $\hat{\nabla} \hat{\Phi}_{k}=\left(\left(1-\delta_{k 0}\right) \delta_{k j}-\delta_{k 0} d^{-\frac{1}{2}}\right)_{j=1, \ldots, d}$. Furthermore, $G[X]=D X^{T} D X$ is the (discrete) metric tensor, where $X$ is the mapping from the reference simplex $\hat{T}$ to the simplex $T$. For the derivatives of the metric tensor we obtain

$$
\begin{aligned}
\partial_{X_{k}} G[X](V) & =\left(\partial_{X_{k}} D X(V)\right)^{T} D X+D X\left(\partial_{X_{k}} D X(V)\right)^{T} \\
\partial_{X_{l}} \partial_{X_{k}} G[X](V, W) & =\left(\partial_{X_{l}} D X(W)\right)^{T}\left(\partial_{X_{k}} D X(V)\right)+\left(\partial_{X_{k}} D X(V)\right)^{T}\left(\partial_{X_{l}} D X(W)\right)
\end{aligned}
$$

for $V, W \in \mathbb{R}^{m}$, where $\partial_{X_{k}} D X(V)=\left(\left(\left(1-\delta_{k 0}\right) \delta_{k j}-\delta_{k 0}\right) V_{i}\right)_{\substack{i=1, \ldots, m \\ j=1, \ldots d}}$ is the derivative of the Jacobian of $X$ with respect to the position of the vertex $X_{k}$. From this we immediately deduce

$$
\begin{aligned}
& \partial_{X_{k}} \sqrt{\operatorname{det} G[x]=} \frac{1}{2} \sqrt{\operatorname{det} G[x]} \operatorname{tr}\left(G[X]^{-1} \partial_{X_{k}} G[X](V)\right), \\
& \partial_{X_{k}}\left(G[x]^{-1}\right)(V)=-G[x]^{-1} \partial_{X_{k}} G[X](V) G[x]^{-1}, \\
& \partial_{X_{l}} \partial_{X_{k}}\left(G[x]^{-1}\right)(V, W)=-G[x]^{-1} \partial_{X_{l}} \partial_{X_{k}} G[X](V, W) G[x]^{-1} \\
&+G[x]^{-1} \partial_{X_{l}} G[X](W) G[x]^{-1} \partial_{X_{k}} G[X](V) G[x]^{-1} \\
&+G[x]^{-1} \partial_{X_{k}} G[X](V) G[x]^{-1} \partial_{X_{l}} G[X](W) G[x]^{-1}, \\
& \partial_{X_{l}} \partial_{X_{k}} \sqrt{\operatorname{det} G[x]}(V, W)=\frac{1}{4} \sqrt{\operatorname{det} G[x]} \operatorname{tr}\left(G[X]^{-1} \partial_{X_{l}} G[X](W)\right) \cdot \operatorname{tr}\left(G[X]^{-1} \partial_{X_{k}} G[X](V)\right) \\
&+ \frac{1}{2} \sqrt{\operatorname{det} G[x]} \operatorname{tr}\left(\partial_{X_{l}}\left(G[x]^{-1}\right)(W) \partial_{X_{k}} G[X](V)\right. \\
&\left.+G[X]^{-1} \partial_{X_{l}} \partial_{X_{k}} G[X](V, W)\right) .
\end{aligned}
$$

Finally, we get the following formulas for the derivative of the mass and stiffness matrix

$$
\begin{aligned}
\partial_{X_{k}} M[X](V)= & \partial_{X_{k}} \sqrt{\operatorname{det} G[x]} \hat{M}, \\
\partial_{X_{l}} \partial_{X_{k}} M[X](V, W)= & \partial_{X_{l}} \partial_{X_{k}} \sqrt{\operatorname{det} G[x]}(V, W) \hat{M}, \\
\partial_{X_{k}} L[X](V)= & \frac{1}{d !}\left(\partial_{X_{k}} \sqrt{\operatorname{det} G[x]}(V) G[x]^{-1} \hat{\nabla} \hat{\Phi}_{i} \cdot \hat{\nabla} \hat{\Phi}_{j}\right. \\
& \left.\quad+\sqrt{\operatorname{det} G[x]} \partial_{X_{k}}\left(G[x]^{-1}\right)(V) \hat{\nabla} \hat{\Phi}_{i} \cdot \hat{\nabla} \hat{\Phi}_{j}\right)_{i, j=0, \ldots, d}, \\
\partial_{X_{l}} \partial_{X_{k}} L[X](V, W)= & \frac{1}{d !}\left(\partial_{X_{l}} \partial_{X_{k}} \sqrt{\operatorname{det} G[x]}(V, W) G[x]^{-1} \hat{\nabla} \hat{\Phi}_{i} \cdot \hat{\nabla} \hat{\Phi}_{j}\right. \\
& \left.+\sqrt{\operatorname{det} G[x]} \partial_{X_{l}} \partial_{X_{k}}\left(G[x]^{-1}\right)(V, W) \hat{\nabla} \hat{\Phi}_{i} \cdot \hat{\nabla} \hat{\Phi}_{j}\right)_{i, j=0, \ldots, d} .
\end{aligned}
$$


Derivatives of the local anisotropic area functional

We restrict ourselves here to the evolution of polygonal curves under discrete Willmore flow and focus on the local area functional on a single line segment connecting points $X_{0}$ and $X_{1}$ in $\mathbb{R}^{2}$. We have $A_{\gamma}[X]=\frac{1}{2} \sqrt{\operatorname{det} G[X]} \gamma(N)$, where $N=\frac{D^{90}\left(X_{1}-X_{0}\right)}{\left|X_{1}-X_{0}\right|}$ denotes the discrete normal and for $d=1$ this time $G[X]=\left|X_{1}-X_{0}\right|$. Due to the 1-homogeneity of $\gamma$ we can rewrite $A_{\gamma}$ and obtain $A_{\gamma}[X]=\frac{1}{2} \tilde{\gamma}\left(X_{1}-X_{0}\right)$ with $\tilde{\gamma}=\gamma \circ D^{90}$. Hence, via straightforward differentiation we obtain

$$
\begin{aligned}
\partial_{X_{i}} A_{\gamma}(V) & =\tilde{\gamma}, z\left(X_{1}-X_{0}\right) \cdot V\left(\delta_{1 i}-\delta_{0 i}\right) \\
\partial_{X_{j}} \partial_{X_{i}} A_{\gamma}(V, W) & =\tilde{\gamma}, z z\left(X_{1}-X_{0}\right) V \cdot W\left(\delta_{1 i}-\delta_{0 i}\right)\left(\delta_{1 j}-\delta_{0 j}\right) \\
\partial_{X_{k}} \partial_{X_{j}} \partial_{X_{i}} A_{\gamma}(V, W, Z) & =\tilde{\gamma}, z z z\left(X_{1}-X_{0}\right)(V, W, Z)\left(\delta_{1 i}-\delta_{0 i}\right)\left(\delta_{1 j}-\delta_{0 j}\right)\left(\delta_{1 k}-\delta_{0 k}\right) .
\end{aligned}
$$

Acknowledgement. Nadine Balzani has been supported by the DFG collaborative research center SFB 611 at Bonn University and the Hausdorff Center for Mathematics.

\section{REFERENCES}

1. Barrett, J. W., Garcke, H., \& Nürnberg, R., A parametric finite element method for fourth order geometric evolution equations, J. Comp. Phys. 222 (2007), 441-467. Zbl1112.65093 MR2298053

2. Bellettini, G., Caselles, V., Chambolle, A., \& Novaga, M., Crystalline mean curvature flow of convex sets. Arch. Ration. Mech. Anal. 179 (2006), 109-152. Zbl1148.53049 MR2208291

3. Bellettini, G., \& Mugnai, L., Anisotropic geometric functionals and gradient flows, Banach Center Publications 86 (2009), 21-43. Zbl1189.53063 MR2571479

4. Ben-Tal, A., \& Nemirovski, A., Robust optimization - methodology and applications, Mathematical Programming 92 (2002), 453-480. Zbl1007.90047 MR1905762

5. Bertalmio, M., Bertozzi, A., \& Sapiro, G., Navier-stokes, fluid dynamics, and image and video inpainting, IEEE Proceedings of the International Conference on Computer Vision and Pattern Recognition, vol. 1, 2001, pp. 355-362.

6. Bertalmio, M., SAPiro, G., Caselles, V., \& BAllester, C., Image inpainting, (2000, ), pp. 417424.

7. Bobenko, A., \& Schröder, P., Discrete Willmore flow, SIGGRAPH (Courses), ACM Press, 2005.

8. Chambolle, A., An algorithm for mean curvature motion, Interfaces and free Boundaries 6 (2004), 195-218. Zbl1061.35147 MR2079603

9. Chambolle, A., \& Novaga, M., Convergence of an algorithm for the anisotropic and crystalline mean curvature flow, SIAM J. Math. Anal. 37 (2006), 1978-1987. Zbl1116. 35074 MR2213402

10. Chan, T. F., Kang, S. H., \& Shen, J., Euler's elastica and curvature-based inpainting, SIAM Appl. Math. 63 (2002), 564-592. Zbl1028.68185 MR1951951

11. Clarenz, U., Enclosure theorems for extremals of elliptic parametric functionals, Calculus of Variations and Partial Differential Equations 15 (2002), 313-324. Zbl1018.53006 MR1938817

12. Clarenz, U., The Wulff-shape minimizes an anisotropic Willmore functional, Interfaces and Free Boundaries 6 (2004), 351-359. Zbl1072.35184 MR2095337

13. Clarenz, U., Diewald, U., Dziuk, G., Rumpf, M., \& Rusu, R., A finite element method for surface restoration with smooth boundary conditions, Computer Aided Geometric Design 21 (2004), 427-445. Zbl1069.65546 MR2058390

14. Deckelnick, K., \& Dziuk, G., Error analysis of a finite element method for the Willmore flow of graphs., Interfaces and Free Boundaries 8 (2006), 21-46. Zbl1102. 35047 MR2231251 
15. Deckelnick, K., \& Dziuk, G., Error analysis for the elastic flow of parametrized curves, Math. Comp. 78 (2009), 645-671. Zbl1198.65183 MR2476555

16. DeCKelnick, K., \& SCHIEWECK, F., Error analysis for the approximation of axisymmetric willmore flow by c1-elements, Tech. Report Preprint Nr. 23/2009, 20 pp., Universität Magdeburg, 2009. Zbl1227. 65079 MR2754216

17. Diewald, U., Anisotrope krümmungsflüsse parametrischer flächen sowie deren anwendung in der flächenverarbeitung, Dissertation, Universität Duisburg-Essen, Campus Duisburg, 2005.

18. Droske, M., On variational problems and gradient flows in image processing, Dissertation, Universität Duisburg-Essen, Campus Duisburg, 2005. Zb11196.94003

19. Droske, M., \& RUMPF, M., A level set formulation for Willmore flow, Interfaces and Free Boundaries 6 (2004), 361-378. Zbl1062.35028 MR2095338

20. DU, Q., LIU, C., \& WANG, X., Simulating the deformation of vesicle membranes under elastic bending energy in three dimensions, Journal of Computational Physics 212 (2006), 757-777. Zbl1086.74024 MR2187911

21. DzIUK, G., Finite elements for the Beltrami operator on arbitrary surfaces, Partial Differential Equations and Calculus of Variations (HildebrandT, S., \& LEIS, R., eds.), Lecture Notes in Mathematics 1357, Springer, 1988, pp. 142-155. Zbl0663.65114 MR0976234

22. DzIUK, G., An algorithm for evolutionary surfaces, Numer. Math. 58 (1991), 603-611. Zb10714. 65092 MR1083523

23. DzIUK, G., Convergence of a semi-discrete scheme for the curve shortening flow, Mathematical Models and Methods in Applied Sciences 4 (1994), 589-606. Zb10811.65112 MR1291140

24. DzIUK, G., Discrete anisotropic curve shortening flow, SIAM Journal on Numerical Analysis 36 (1999), 1808-1830. Zbl0942.65112 MR1712165

25. DzIUK, G., Computational parametric Willmore flow, Numerische Mathematik 111 (2008), 55-80. Zb11158.65073 MR2448203

26. Dziuk, G., Kuwert, E., \& Schätzle, R., Evolution of elastic curves in $\mathbb{R}^{n}$ : existence and computation, SIAM J. Math. Anal. 33, no. 5 (2002), 1228-1245 (electronic). Zbl1031. 53092 MR1897710

27. Franken, M., RumpF, M., \& Wirth, B., A phase field based pde constraint optimization approach to time discrete willmore flow, International Journal of Numerical Analysis and Modeling (2011), accepted.

28. HASlinger, J., \& MÄKInEN, R. A. E., Introduction to shape optimization: theory, approximation, and computation, Society for Industrial and Applied Mathematics (SIAM), Philadelphia, PA, USA, 2003. Zbl1020.74001 MR1969772

29. HelfRICH, W., Elastic properties of lipid bilayers: Theory and possible experiments, Zeitschrift für Naturforschung 28c (1973), 693-703.

30. Hinze, M., Pinnau, R., Ulbrich, M., \& Ulbrich, S., Optimization with PDE Constraints, Mathematical Modelling: Theory and Applications, vol. 23, Springer, 2009. Zbl1167.49001 MR2516528

31. Kuwert, E., \& Schätzle, R., The Willmore flow with small initial energy, J. Differential Geom. 57 (2001), 409-441. Zbl1035.53092

32. Kuwert, E., \& Schätzle, R., Gradient flow for the Willmore functional, Comm. Anal. Geom. 10 (2002), 307-339. Zbl1029.53082 MR1900754

33. Kuwert, E., \& Schätzle, R., Removability of point singularities of Willmore surfaces, Annals of Mathematics (2) 160 (2004), 315-357. Zbl1078. 53007 MR2119722

34. Luckhaus, S., \& Sturzenhecker, T., Implicit time discretization for the mean curvature flow equation, Calc. Var. 3 (1995), 253-271. Zb10821. 35003 MR1386964

35. Mayer, U., \& Simonett, G., A numerical scheme for axisymmetric solutions of curvature driven free boundary problems with applications to the Willmore flow, Interphases and Free Boundaries 4 (2002), 89-109. Zbl1005.65095 MR1877537

36. Mumford, D., Elastica and computer vision, Algebraic Geometry and Its Applications (BAJAJ, C., ed.), 
Springer, New York, 1994, pp. 491-506. Zb10798.53003 MR1272050

37. Nemitz, O., Anisotrope Verfahren in der Bildverarbeitung: Gradientenflüsse, Level-Sets und Narrow Bands, Dissertation, Rheinische Friedrich-Wilhelms-Universität Bonn, 2008.

38. Nitzberg, M., Mumford, D., \& Shiota, T., Filtering, Segmentation and Depth (Lecture Notes in Computer Science Vol. 662), Springer-Verlag Berlin Heidelberg, 1993. Zb10801.68171 MR1226232

39. Nocedal, J., \& Wright, S. J., Numerical Optimization, Springer, New York/Berlin, 1999. Zb10930. 65067 MR1713114

40. Olischläger, N., \& Rumpf, M., Two Step Time Discretization of Willmore Flow, Proceedings of the 13th IMA International Conference on Mathematics of Surfaces XIII (Berlin, Heidelberg), SpringerVerlag, 2009, pp. 278-292.

41. Palmer, B., Variational Problems which are Quadratic in the Surface Curvatures, AIP Conference Proceedings: Curvature and Variational Modeling in Physics and Biophysics, vol. 1002, 2007, pp. 33-70. MR2483891

42. Palmer, B., Equilibria for anisotropic bending energies, Journal of Mathematical Physics 50 (2009). Zb11202. 49055 MR2494852

43. Polden, A., Closed Curves of Least Total Curvature, SFB 382 Tübingen, Preprint 13 (1995).

44. Polden, A., Curves and Surfaces of Least Total Curvature and Fourth-Order Flows, Dissertation, Universität Tübingen (1996).

45. PozZI, P., Anisotropic mean curvature flow in higher codimension, PAMM 8 (1) (2008), 10849-10850.

46. RIVIÈre, T., Analysis aspects of Willmore surfaces, Inventiones mathematicae (2008), 1-45. Zbl1155. 53031 MR2430975

47. RUSu, R., An algorithm for the elastic flow of surfaces, Interfaces and Free Boundaries 7 (2005), 229239. Zbl1210.35149 MR2171130

48. Seifert, U., Configurations of fluid membranes and vesicles, Advances in Physics 46 (1997), 13-137.

49. Simonett, G., The Willmore Flow near spheres, Diff. and Integral Eq. 14(8) (2001), 1005-1014. Zbl1161.35429 MR1827100

50. Welch, W., \& Witkin, A., Variational surface modeling, SIGGRAPH Computer Graphics, vol. 26, 1992, pp. 157-166.

51. Willmore, T., Riemannian Geometry, Claredon Press, Oxford, 1993. Zb10797.53002 MR1261641

52. XU, G., \& PAN, Q., G ${ }^{1}$ surface modelling using fourth order geometric flows, Computer-Aided Design 38 (2006), 392-403.

53. Yoshizawa, S., \& Belyaev, A. G., Fair triangle mesh generation with discrete elastica, Proceedings of the Geometric Modeling and Processing; Theory and Applications (GMP'02) (Washington, DC, USA), IEEE Computer Society, 2002, pp. 119-123. 